\title{
Voltage-dependent gating of hERG potassium channels
}

\author{
Yen May Cheng and Tom W. Claydon* \\ Department of Biomedical Physiology and Kinesiology, Simon Fraser University, Burnaby, BC, Canada
}

Edited by:

Gildas Loussouarn, University of

Nantes, France

Reviewed by:

Zeineb Es-Salah-Lamoureux, INSERM, France

Medha M. Pathak, University of California Irvine, USA

*Correspondence:

Tom W. Claydon, Department of Biomedical Physiology and Kinesiology, Simon Fraser University, 8888 University Drive, Burnaby, BC, Canada V5A 156

e-mail: thomas_claydon@sfu.ca
The mechanisms by which voltage-gated channels sense changes in membrane voltage and energetically couple this with opening of the ion conducting pore has been the source of significant interest. In voltage-gated potassium (Kv) channels, much of our knowledge in this area comes from Shaker-type channels, for which voltage-dependent gating is quite rapid. In these channels, activation and deactivation are associated with rapid reconfiguration of the voltage-sensing domain unit that is electromechanically coupled, via the S4-S5 linker helix, to the rate-limiting opening of an intracellular pore gate. However, fast voltage-dependent gating kinetics are not typical of all Kv channels, such as Kv11.1 (human ether-à-go-go related gene, hERG), which activates and deactivates very slowly. Compared to Shaker channels, our understanding of the mechanisms underlying slow hERG gating is much poorer. Here, we present a comparative review of the structure-function relationships underlying activation and deactivation gating in Shaker and hERG channels, with a focus on the roles of the voltage-sensing domain and the S4-S5 linker that couples voltage sensor movements to the pore. Measurements of gating current kinetics and fluorimetric analysis of voltage sensor movement are consistent with models suggesting that the hERG activation pathway contains a voltage independent step, which limits voltage sensor transitions. Constraints upon hERG voltage sensor movement may result from loose packing of the S4 helices and additional intra-voltage sensor counter-charge interactions. More recent data suggest that key amino acid differences in the $h E R G$ voltage-sensing unit and S4-S5 linker, relative to fast activating Shaker-type Kv channels, may also contribute to the increased stability of the resting state of the voltage sensor.

Keywords: hERG, gating, voltage sensor, gating charge transfer center, potassium channel, S4-S5 linker, Shaker

\section{INTRODUCTION OVERVIEW}

The finely tuned voltage-dependence of ion channels has long been a topic of interest for ion channel biophysicists. For voltagegated $\mathrm{K}^{+}(\mathrm{Kv})$ channels, much of the knowledge gathered to date on voltage-dependent gating has focused on structure-function relationships in the archetypal Shaker channel and other closely related members of the Kv1 family. However, the fast voltagedependent activation (opening) and deactivation (closing) gating kinetics of Shaker are not typical of all Kv channels. For example, Kv11.1 (human ether-à-go-go related gene, hERG) channels activate and deactivate very slowly. hERG channels underlie the cardiac delayed rectifier current, $I_{\mathrm{Kr}}$, and their unique voltagedependent gating properties are critical to normal repolarization during the cardiac action potential. This highlights the importance of understanding the processes involved in hERG function. However, compared to Shaker-like channels, the processes regulating the gating of hERG channels are much less clear. This review focuses on the molecular determinants of the unique voltagedependent activation and deactivation kinetics of hERG channels. To begin, the rest of this section provides a brief review of general $\mathrm{Kv}$ channel structure and function and an introduction to the differences between hERG and Shaker gating kinetics. The following sections will then focus on the roles of the S4 voltage sensor and S4-S5 linker in the slow gating of hERG channels. As part of this discussion potentially important structural differences between hERG and Shaker channels within these regions will be highlighted.

\section{BASIC Kv CHANNEL ARCHITECTURE}

In their simplest form, $\mathrm{Kv}$ channels consist of a tetrameric assembly of identical $\alpha$-subunits around a central pore. Each $\alpha$-subunit has six $\alpha$-helical transmembrane segments, S1-S6, and cytoplasmic Nand C-terminal domains (Figure 1A). The voltage-sensing domain of each $\alpha$-subunit is comprised of segments S1-S4. Importantly, the S4 segment has the distinction of containing four to eight basic residues (Arg or Lys), spaced apart by pairs of hydrophobic residues, that allow it to act as the primary voltage sensor. The S1-S3 segments, which possess a number of negatively charged residues (Papazian et al., 1987), complete the voltage-sensing domain and are thought to help stabilize the positively charged S4 segment in the lipid bilayer and to define the pathway for its movement through the electric field (reviewed in Börjesson and Elinder, 2008; Chanda and Bezanilla, 2008; Catterall, 2010).

The pore domain of an $\alpha$-subunit is formed by the S5 and S6 segments, which are joined by a re-entrant pore helix and loop (P-loop). The X-ray crystal structures of selected bacterial and eukaryotic Kv channels have been solved (Jiang et al., 2003; Long et al., 2005a, 2007; Chen et al., 2010) and show that the pore domains are arranged with four-fold symmetry around the ion conduction pathway, while the voltage-sensing domains lie at the periphery (Figure 1B; Long et al., 2005a; Chen et al., 2010).The 

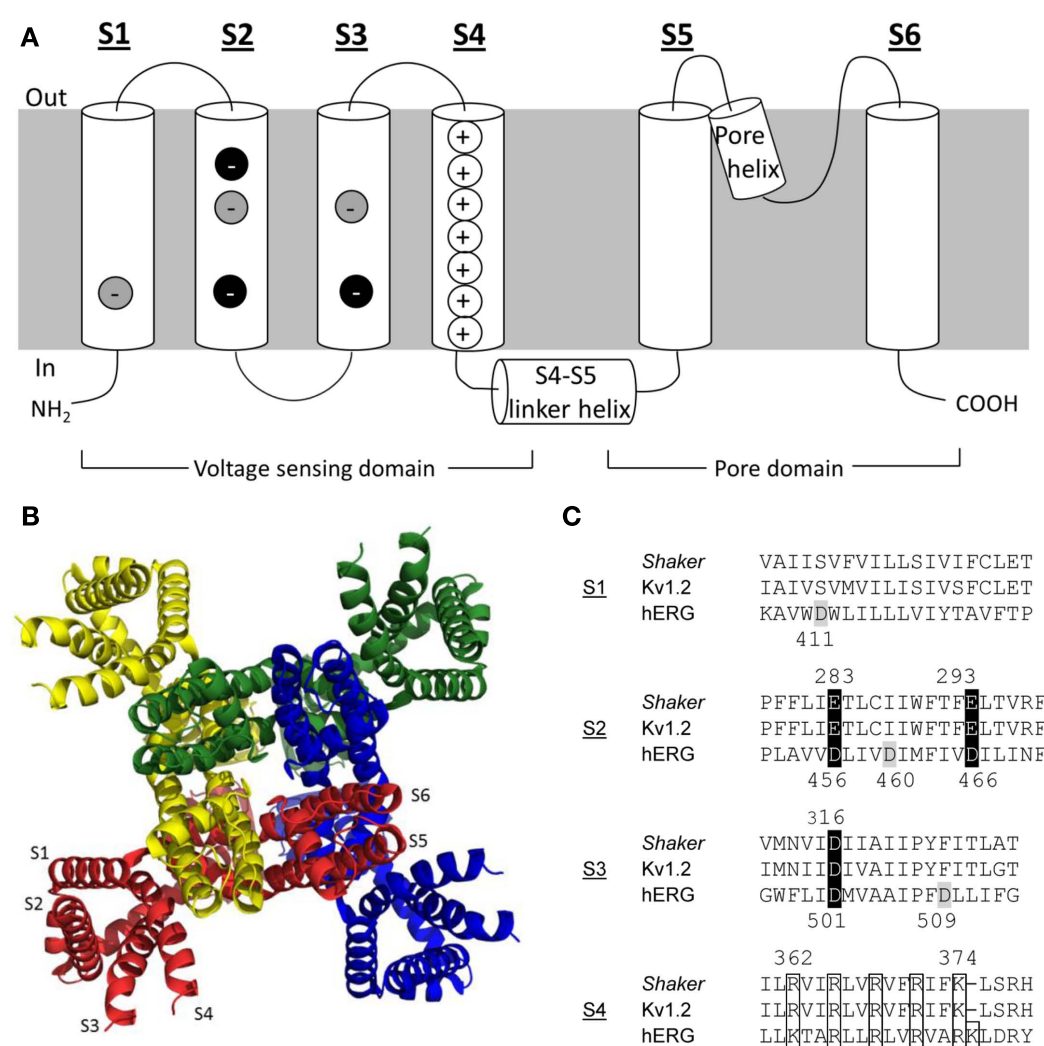

C

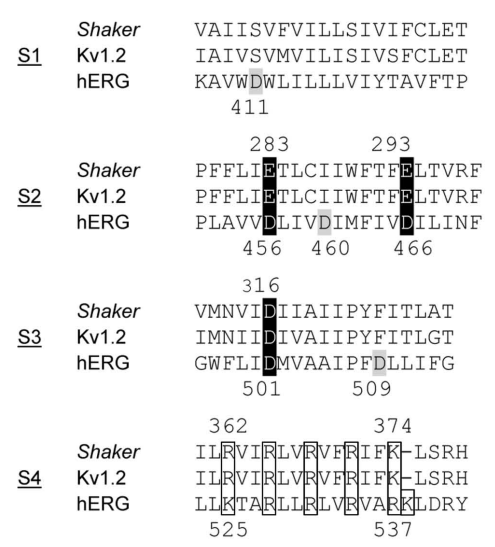

FIGURE 1 | Kv channel structure. (A) Cartoon representation of the membrane topology of a Kv channel $\alpha$-subunit. Key charged residues within the voltage-sensing domain are highlighted. Negative charges highlighted in black are highly conserved, whereas those in gray are conserved only in the eag channel family. (B) Ribbon representation of the Kv channel tetrameric assembly based on the Kv1.2 crystal structure
(Long et al., 2005a). Each subunit has been highlighted a different color for clarity. The model shows a view from the top of the channel looking down along the permeation pathway (at center). (C) Alignments of primary S1-S4 sequences in Shaker, Kv1.2, and hERG channels. Key charged residues are highlighted with the same color scheme as in panel A. crystal structures of bacterial KcsA and MthK channels (Doyle et al., 1998; Jiang et al., 2002a,b) suggested that the convergence of the bottom of the M2 helices (analogous to S6 in eukaryotic Kv channels) at a "bundle crossing" forms an activation gate to control access between the pore and the cytoplasm. A conserved glycine residue has been proposed to serve as the "hinge" that allows the activation gate to open and close (Jiang et al., 2002a). Eukaryotic channels typically also have a PxP motif that lies distal to the glycine hinge, which is thought to introduce a sharp bend in the S6 helix that may contribute to opening and closing of the activation gate (del Camino et al., 2000; del Camino and Yellen, 2001) and/or coupling of the pore to the voltage-sensing domain (Lu et al., 2002; Long et al., 2005b). Although the X-ray crystal structures present only a static view and cannot alone show the dynamics of $\mathrm{Kv}$ channel gating, they have provided the structural framework necessary to describe the processes underlying $\mathrm{Kv}$ channel gating in response to changes in membrane voltage.

\section{THE ELECTROMECHANICAL BASIS OF VOLTAGE-DEPENDENT Kv CHANNEL GATING}

The culmination of Kv channel activation in response to depolarization is the opening of the intracellular gate allowing passage of $\mathrm{K}^{+}$ions through the channel pore. Subsequent membrane repolarization causes channel deactivation and the closure of the activation gate. It is instructive to consider the electromechanical processes of activation and deactivation in two parts, where (i) the change in membrane potential is first "sensed" by the channel and then (ii) translated into opening or closing of the activation gate.

Prior to any structural information, Hodgkin and Huxley (1952) proposed that voltage-dependent $\mathrm{K}^{+}$and $\mathrm{Na}^{+}$channels possessed four charged particles that resided in the membrane electric field and served as voltage sensors, moving between activated and deactivated positions in response to changes in voltage. Membrane depolarization increased the probability that all four voltage sensors would be in the activated position, which was necessary for channel opening. When any of the sensors were in a deactivated position, the channel would be in one of several possible closed states. Evidence in support of this model was provided some 22 years later by measurements of the movement of gating charges across the membrane electric field, showing a capacitive gating current associated with channel activation (e.g., Armstrong and Bezanilla, 1974; Gilly and Armstrong, 1980). The S4 transmembrane segment was proposed to serve as the voltage sensor when it was found that $\mathrm{Kv}$ channel $\alpha$-subunits and Nav 
channel domains contain a conserved sequence of repeating positively charged residues (Noda et al., 1984; Papazian et al., 1987). While the role of S4 as the primary voltage sensor for Nav and $\mathrm{Kv}$ channels is now widely accepted, the dynamics of sensor movement through the electric field, which trigger opening and closing of channel, remain to be precisely defined.

Three general models of voltage-dependent S4 movement have been proposed: (i) the helical-screw or sliding helix model, where S4 rotates along its axis while being translated across the membrane to move gating charges across the electric field (Durell et al., 1998; Keynes and Elinder, 1999); (ii) the transporter-like model, where a mostly rotational movement of S4 (and perhaps a rearrangement of the membrane) essentially flips $\mathrm{S} 4$ charges from one side of the membrane to the other (Papazian and Bezanilla, 1997), and; (iii) the paddle model, initially based on the crystal structure of KvAP, in which a voltage sensor paddle comprised of $\mathrm{S} 4$ and the C-terminal half of S3 form a rigid structure that moves through the membrane and in close proximity to S1 and S2 (Jiang et al., 2003). While there has been significant debate over which model is most accurate, in recent years the models show some consensus. These models suggest that the series of gating charges is moved $6-15 \AA$ across the membrane by S4 rotation and translation. A more detailed discussion concerning models of voltage sensor movement can be found in several recent reviews (Börjesson and Elinder, 2008; Chanda and Bezanilla, 2008; Catterall, 2010).

Movement of the voltage-sensing unit in response to changes in membrane potential is translated into the concerted opening or closing of the activation gate within the pore domain. Solution of the high resolution Kv1.2 crystal structure shows that within the same $\alpha$-subunit the voltage-sensing domain lies away from the pore domain (Figure 1B), such that the top of S4 is actually in closer proximity with the pore domain of a neighboring subunit (Long et al., 2005a; Chen et al., 2010). On the cytoplasmic face of the channel the $\alpha$-helical S4-S5 linker appears to lie across the distal S6 helix below the PxP motif within the same subunit, suggestive of a possible coupling mechanism between the two domains. Such structural observations are consistent with functional studies showing that specific interactions between the
S4-S5 linker and the lower S6 segment are critical for voltagedependent gating (e.g., Chen et al., 2001; Ding and Horn, 2002, 2003; Lu et al., 2002; Tristani-Firouzi et al., 2002; Labro et al., 2008; Nishizawa and Nishizawa, 2009). In the light of this evidence, it is proposed that the outward movement of $\mathrm{S} 4$ upon depolarization may create tension in the S4-S5 linker, which would allow the C-terminal end of S6 to pivot away from the central axis of the pore to open the activation gate. Conversely, repolarization and inward movement of S4 would tend to push the S4-S5 linker radially inward and exert a force on the internal end of $\mathrm{S} 6$ so that the activation gate closes.

\section{hERG CHANNELS HAVE UNIQUELY SLOW ACTIVATION AND DEACTIVATION KINETICS}

Compared to most other Kv channels, hERG channel gating kinetics are unusually slow (Figure 2). While archetypal Shaker channels activate with time constants of a few milliseconds, hERG channels activate and deactivate over hundreds of milliseconds (Sanguinetti and Tristani-Firouzi, 2006). Voltage-clamp fluorimetry (VCF) and gating current data have been purported to show that hERG slow activation and deactivation are due, at least in part, to rate-limiting voltage sensor movement (Smith and Yellen, 2002; Piper et al., 2003). In stark contrast, S4 movement in Shaker and other Kv channels is very rapid (e.g., Bezanilla et al., 1994; Zagotta et al., 1994b; Mannuzzu et al., 1996; Cha and Bezanilla, 1997; Loots and Isacoff, 1998; Kanevsky and Aldrich, 1999) and the rate-limiting step for channel opening and closing lies further along the activation pathway and most likely involves the final concerted opening (or closing) of the activation gate (Ledwell and Aldrich, 1999; Bezanilla, 2000; Fedida and Hesketh, 2001). The comparatively slower voltage sensor movement in hERG channels points to additional barriers or constraints to outward S4 movement that must be overcome before hERG channels can open.

In addition to putative slow $S 4$ movement, there may be other mechanisms underlying slow hERG activation and deactivation gating. For example, we have shown that electromechanical coupling between the voltage-sensing domain and the pore domain by the S4-S5 linker helix is less efficient in hERG when compared

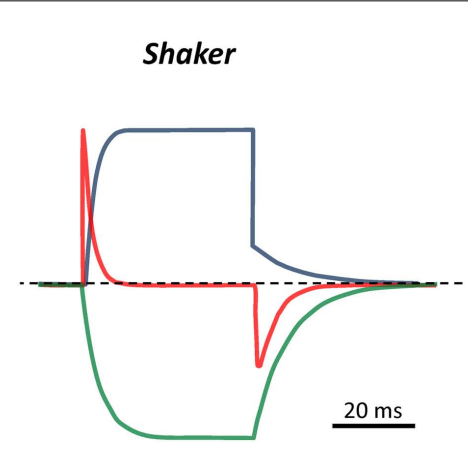

FIGURE 2 | Comparison of gating and kinetics in Shaker and hERG channels. Cartoon representation of ionic current, gating current and fluorescence reports for Shaker (fast-inactivation removed) and hERG channels in response to a depolarizing step from -80 to $0 \mathrm{mV}$, followed by a repolarizing step to $-60 \mathrm{mV}$. Fluorescence traces represent reports from

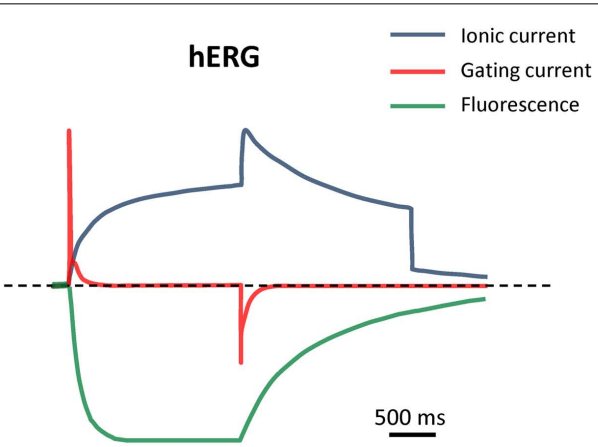

fluorophores attached at the outer end of S4, to Shaker A359C or hERG L520C. Note the different timescales and step durations for the fast-activating Shaker and slow-activating hERG channels. Schematics were generated based on data from primary sources cited in the text (see Does Slow S4 Voltage Sensor Movement Underlie Slow hERG Gating Kinetics?"). 
to Shaker channels (e.g., Van Slyke et al., 2010) and this could contribute to slow responses to changes in membrane potential. hERG deactivation also appears to be more complex than that of Shaker channels, in that additional modulation of deactivation may come from the interaction of an N-terminal Per-Arnt-Sim (PAS) domain with other regions of the channel, perhaps the S4S5 linker and/or the C-terminus (Morais Cabral et al., 1998; Wang et al., 1998; Gustina and Trudeau, 2009, 2011; Fernández-Trillo et al., 2011).

Following activation, and with continued depolarization, many $\mathrm{Kv}$ channels undergo an additional gating process of inactivation where they enter into a non-conducting inactivated state that is distinct from the closed state occupied at negative potentials. Typically, inactivation is voltage-independent and relatively slow compared to channel activation, such that significant outward current may be observed before inactivation is observed as a decay in current (reviewed in Kurata and Fedida, 2006). hERG inactivation is unique in that both its onset and recovery $(1-10 \mathrm{~ms}$ timecourses) are much faster than the kinetics for activation and deactivation (Wang et al., 1997). This is important for its physiological role conducting repolarizing current during the late phase of the cardiac action potential (Sanguinetti and Tristani-Firouzi, 2006). In further contrast to inactivation in channels such as Shaker, hERG inactivation also appears to depend directly on the membrane potential and the S4 segment has been proposed to serve as the voltage sensor for both the inactivation and activation processes (Piper et al., 2003). A thorough discussion of the voltagedependent features of hERG (and other $\mathrm{Kv}$ channel) inactivation is beyond the scope of this review, and will not be discussed in further detail here.

In sum, compared to other members of the $\mathrm{K}^{+}$channel superfamily, hERG channels exhibit uniquely slow activation and deactivation kinetics. The remainder of this review turns attention to the structure-function relationships underlying these slow gating kinetics, with particular focus on the interactions involving the charged S4 voltage sensor and the S4-S5 linker helix that couples the voltage-sensing domain to the pore. A comparison between these structures in hERG and Shaker channels will be presented, along with a discussion of the extent to which differences in the amino acid sequences of these regions account for the differences in the voltage-dependent gating kinetics of these two channel types. Finally, the question of whether a gating charge transfer center like that recently described for Shaker channels is present in hERG channels is addressed.

\section{REGULATION OF VOLTAGE SENSOR MOVEMENT IN hERG AND SHAKER CHANNELS IDENTITY AND LOCATION OF CHARGE-CARRYING RESIDUES IN THE VOLTAGE-SENSING DOMAIN}

As we do not yet have X-ray crystal structures for hERG or Shaker channels, much of the structural information for these and other $\mathrm{Kv}$ channels derives from comparisons of the primary sequences and alignment with the Kv1.2 X-ray crystal structures (Long et al., 2005a; Chen et al., 2010). Based on the high sequence homology between Kv1.2 and Shaker channels, as well as the Kv1.2 crystal structure, the S4 helix in Shaker contains six basic residues (R1R4, K5, R6; Figure 1), consistent with earlier predictions (Papazian et al., 1987). Early mutagenesis studies (Liman et al., 1991; Papazian et al., 1991) had shown that neutralization of R1-K5 resulted in a reduced voltage-dependence of activation, which suggested that they, and consequently the S4 segment, were involved in voltage sensing. Direct measurements of gating charge from gating current experiments in Shaker have shown that channel opening is associated with the movement of $\sim 12-13 \mathrm{e}_{0}$ charges across the electric field (Schoppa et al., 1992; Aggarwal and Mackinnon, 1996; Noceti et al., 1996; Seoh et al., 1996). Gating current measurements from charge neutralization mutants confirmed that the gating charge associated with activation was indeed carried by the S4 charges, with the largest contributions made from the four outermost arginine residues (R1-R4) and perhaps a smaller contribution from K5 (Aggarwal and Mackinnon, 1996; Seoh et al., 1996). The potential contributions to the gating charge of three negatively charged residues in S2 (E283, E293) and S3 (D316) that are highly conserved among Kv channels (Figure 1A,C) have similarly been assessed; with a significant contribution coming from E293 in S2 and a much smaller contribution from D316 (Seoh et al., 1996).

In addition to these charge neutralization studies, other studies were conducted to determine the extent of S4 translocation across the membrane. Some used the substituted cysteine accessibility method, which involves mutating a specific residue to a cysteine and determining its state-dependent accessibility to externally or internally applied cysteine-reactive compounds such as methanethiosulfonate (MTS) reagents or $p$ chloromercuribenzenesulfonate (pCMBS). After accounting for methodological differences, the results of these studies (Larsson et al., 1996; Yusaf et al., 1996; Baker et al., 1998; Wang et al., 1999) suggested that the $\mathrm{S} 4$ charged residues reside in an intracellularly accessible water-filled crevice at negative resting potentials and that depolarization results in the first two $(\mathrm{R} 1, \mathrm{R} 2)$ and perhaps the third (R3) positively charged residues moving to an externally accessible position. In a different approach, Starace et al. (1997), Starace and Bezanilla (2001), Starace and Bezanilla (2004) substituted the S4 charges with histidine residues and determined their accessibility by examining the state-dependent titration of gating charge by intra- and extracellular $\mathrm{pH}$. The results of these studies also suggested that at resting potentials, the Shaker S4 charges reside in a narrow, intracellularly accessible water-filled crevice and membrane depolarization results in the translocation of R1-R3, and most likely R4, but not K5 or R6, across the membrane to an extracellularly accessible crevice (Starace et al., 1997; Starace and Bezanilla, 2001). Compared to the substituted cysteine accessibility method, the potentially charge-conserving nature of histidine substitutions may contribute to a more native conformation of the voltage-sensing domain, as it has been reported that cysteine substitutions can affect the S4 topology such that the membrane spanning portion is reduced (Wang et al., 1999). Intriguingly, histidine substitution at $\mathrm{R} 1$ or $\mathrm{R} 4$ resulted in the formation of a proton pore at hyperpolarized and depolarized potentials, respectively (Starace and Bezanilla, 2001, 2004). This suggests that the substituted histidine residue at position 1 and 4 bridges a very narrow gap between the external and internal crevices in the resting and activated states, respectively (Starace and Bezanilla, 2001, 2004). Compared to the substituted cysteine accessibility method, 
the ability of the histidine scanning mutagenesis to show translocation of R3 and R4 likely arises from the small size and improved mobility of protons relative to MTS and pCMBS. To sum, activation of Shaker channels results in the translocation of R1-R3 and probably R4 across the membrane, consistent with the conclusion from gating current measurements showing that much of the gating charge is carried by these four residues (reviewed in detail in Bezanilla, 2000; Catterall, 2010).

The primary sequence homology of hERG channels with Shaker and Kv1.2 channels is considerably poorer than that observed within the Kv1 channel family (Figure 1C). There has been little consensus on how the sequences of hERG and Shaker channels should be aligned and, thus, how to define exactly where the transmembrane segment borders (i.e., extracellular and intracellular limits) are in hERG channels. Within the voltage-sensing domain of hERG, the S4 segment has, depending on the alignment used, up to seven basic residues (K1, R2-R5, K6, R7), although the repeating sequence of a positive residue at every third position separated by two hydrophobic residues capitulates after the fifth arginine residue (Figure 1C). The amount of gating charge moved with hERG activation has been estimated to be $\sim 6 \mathrm{e}_{0}$ using limiting slope analysis of the voltage-dependence of channel open probability $\left(P_{\mathrm{o}}\right)$; even after correction to $8 \mathrm{e}_{0}$ by factoring in the underestimation of total charge by the limiting slope method (Zhang et al., 2004), this is still less than the $\sim 12 \mathrm{e}_{0}$ observed for Shaker. Cysteine substitution of only the three outermost hERG S4 positive charges $(\mathrm{K} 1, \mathrm{R} 2, \mathrm{R} 3)$ resulted in significant decreases in the total gating charge, which suggests that they transfer most of the charge moved during activation (Zhang et al., 2004), compared to the first four charges in Shaker (see above). Similar to E293 in Shaker, hERG D466 in S2 also appears to have a role in gating charge transfer (Zhang et al., 2004). The contributions of the other two conserved negative residues in S2 (D456) and S3 (D501), as well as three additional non-conserved negative charges in the voltage-sensing domain (Liu et al., 2003) to gating charge have not been assessed. To account for the $\sim 1 \mathrm{e}_{0}$ per subunit difference in gating charge between hERG and Shaker, Zhang et al. (2004) aligned the first positive charge in hERG (K1; i.e., K525) with the second positively charged residue in Shaker (R2; i.e., R365) and rKv1.2 (R297). This was supported by the finding that hERG K1 but not R2 was accessible to externally applied MTSET (Subbiah et al., 2004; Zhang et al., 2004), which the authors interpreted to be consistent with similar experiments showing inaccessibility of the equivalent Shaker R3 residue to external MTSET (Larsson et al., 1996; Baker et al., 1998). A recent study using ROSETTA modeling and molecular dynamics to generate a hERG structural model based on the available Kv1.2, KvAP, and Kv1.2-Kv2.1 chimera Xray crystal structures is also supportive of this hERG K1:Shaker R2 alignment (Subbotina et al., 2010).

The S4 alignment for hERGK1 and Shaker R2 presented by Zhang et al. (2004) had been proposed before, based on the results of alignment software (Liu et al., 2003; Subbiah et al., 2004), and has since been used by others to interpret their findings (Subbiah et al., 2005; Zhang et al., 2005; Es-Salah-Lamoureux et al., 2010). As alluded to above, however, an alternative alignment where hERG K1 aligns with Shaker R1 has also frequently been used (Wang et al., 1997; Smith and Yellen, 2002; Silverman et al., 2003; Piper et al., 2005; Elliott et al., 2009), based primarily on computergenerated alignments of the hERG and Shaker sequences. Support for this second alignment comes from a recent report that a stretch of hERG S4 residues (523-529) encompassing the two outermost positive charges (K525, R528) becomes accessible to external pCMBS with depolarization (Elliott et al., 2009). The region of external pCMBS accessible Shaker S4 residues (I360-L366) also includes the first two charged residues (R362, R365; Yusaf et al., 1996), which suggests that the extent of S4 movement is very similar in both hERG and Shaker channels. These results, along with the finding that both hERG L523 and Shaker I360 correspond to the interface between the extracellular solution and the cell membrane at rest (Wang et al., 1999; Elliott et al., 2009), support an alignment of hERG K1 with Shaker R1. Furthermore, comparison of the shifts in the voltage-dependence of activation caused by cysteine substitution along the hERG and Shaker S4 segments also agrees with a hERG K1:Shaker R1 alignment (Elliott et al., 2009). The disagreement of these results obtained with pCMBS with those showing that hERG R528C was inaccessible to MTSET (Zhang et al., 2004) were reconciled by demonstrating that MTSET can react with externally accessible cysteines in hERG and Shaker without producing an appreciable effect on channel function and may therefore give a less reliable report on cysteine accessibility (Wang et al., 1999; Elliott et al., 2009). A consequence of a hERG $\mathrm{K} 1$ :Shaker $\mathrm{R} 1$ alignment is that the reasons for the $4 \mathrm{e}_{0}$ difference in gating charge between the two channels remains unclear.

\section{DOES SLOW S4 VOLTAGE SENSOR MOVEMENT UNDERLIE SLOW hERG GATING KINETICS?}

In the previous section, we have discussed the structure of the voltage-sensing domain and showed that the locations of the charge-carrying residues in the hERG voltage-sensing domain are similar to those for Shaker and Kv1.2 channels. Furthermore, the extent of S4 translocation across the membrane with depolarization appears to be comparable in each of these channels. Thus, gross structural differences between the voltage-sensing domains are unlikely to be the underlying reason for the much slower activation and deactivation kinetics of hERG compared to Shaker channels. We now turn attention toward the rate at which the S4 voltage sensor moves in response to changes in membrane potential. VCF measurements on hERG S4 movement were first performed a decade ago when three consecutive residues (E518, E519, and L520) in the extracellular S3-S4 linker were individually mutated to cysteines and labeled with a fluorescent probe (tetramethylrhodamine-5-maleimide, TMRM); changes in the fluorescent report from these positions were correlated with the voltage- and time-dependence of the ionic current to provide a "picture" of S4 movement (Smith and Yellen, 2002). Fluorophores attached to E518 and E519 showed both fast and slow voltagedependent changes in fluorescence, while L520 gave a single slow report (Figure 2). The slow fluorescence report from all three positions correlated well with the voltage-dependent and temporal properties of ionic current activation and deactivation and responded in parallel to maneuvers that altered the kinetics of channel deactivation. In Shaker channels, fluorescent reports from similar positions indicated that S4 movement occurs prior to and at lower potentials than channel opening (Mannuzzu et al., 1996; 
Cha and Bezanilla, 1997; Loots and Isacoff, 1998). The overlap of the voltage- and time-dependence of the hERG slow fluorescence and ionic current signals suggested that S4 movement reported by the probe is slow and likely the rate-limiting step in the activation process (Smith and Yellen, 2002). Although the fast fluorescence report from probes at E518 and E519 had kinetics and a voltage-dependence that were well correlated with those of channel inactivation, manipulations that affected inactivation did not alter the fast fluorescence (Smith and Yellen, 2002) suggesting that the fast fluorescence component is unlikely to represent a direct report on inactivation gating dynamics.

Shortly after Smith and Yellen's (2002) VCF measurements, the first hERG gating currents were recorded and showed that the kinetics of the movement of the majority of gating charge were remarkably slower $(\tau \approx 50 \mathrm{~ms})$ than that reported for other $\mathrm{Kv}$ channels, such as Shaker (Figure 2). Thus, these electrical measurements supported the conclusion from the fluorescence measurements that slow $\mathrm{S} 4$ movement underlies hERG gating kinetics. hERG gating currents are unique among those of $\mathrm{Kv}$ channels in that they have two components: (i) an initial very rapid transient $(\tau \approx 0.5 \mathrm{~ms})$ and (ii) a slowly decaying component $(\tau \approx 50 \mathrm{~ms})$ that carries the majority of the gating charge (Figure 2; Piper et al., 2003). In contrast to the VCF data, which suggested that S4 movement occurred with a similar voltage-dependence to channel activation, the voltage-dependence of total gating charge $(Q-V)$ movement was left-shifted relative to the conductance-voltage $(G-V)$ relationship. This is more in line with $Q-V$ and $G-V$ relationships previously described for Shaker channels (Bezanilla, 2000) and would appear to indicate that the majority of hERG gating charge movement precedes channel opening. Interestingly, the $Q-V$ for the fast component was right-shifted compared to the $G-$ $V$ and very similar to the fluorescence-voltage relationship for the fast fluorescence component reports from E518 and E519 (Smith and Yellen, 2002). Also consistent with the reported fluorescence data, the fast gating charge component did not correlate well with hERG inactivation properties (other than its kinetics), indicating that the fast gating charge movement did not reflect charge movement associated with hERG inactivation. Instead, based on the observation that the fast gating charge component contributed to the majority of charge moved at potentials below the activation threshold, Piper et al. (2003) concluded that the fast gating charge transient corresponded to transitions between closed states early in the activation pathway. In sum, the results of early VCF and gating current experiments in hERG channels suggested that gating charge and S4 movement occurs prior to channel opening, but that it is slow and therefore contributes to the slow activation process characteristic of hERG.

The view that the slow fluorescence reports from E518C, E519C, and L520C is correlated to S4 movement during activation has recently been questioned. It had previously been reported that labeling of wild-type (WT) hERG with TMRM did not result in any voltage-dependent changes in fluorescence, which was interpreted to indicate that native cysteine residues did not contribute to the fluorescence observed from the mutant channels (Smith and Yellen, 2002; Van Slyke et al., 2010). Es-Salah-Lamoureux et al. (2010) found that TMRM binding to two native cysteine residues in the S1-S2 linker resulted in fluorescence reports that modified the report from E519C in the S3-S4 linker. When the native cysteine residues were substituted with valine, the fluorescence report from E519C was augmented and the fast fluorescence report had a voltage-dependence $(F-V)$ that was left-shifted from the $G-V$ relationship and consistent with the voltage-dependence of total gating charge in hERG movement (Piper et al., 2003). The authors proposed that the fast fluorescence component from E519C reports on rapid S4 movement while the slow component reports on subsequent rearrangements associated with pore opening and closing. Instead, it may be that the fast fluorescence change from E519C reports on the early rapid gating charge movements reported by Piper et al. (2003). Further investigation is required to determine precisely which reconfigurations of the channel the VCF measurements from the hERG S3-S4 linker are reporting on.

\section{hERG ACTIVATION MAY INVOLVE A RATE-LIMITING STEP THAT IS VOLTAGE-INDEPENDENT}

In addition to the results of VCF and gating current experiments that suggest the rate of hERG activation is limited by slow S4 movement, analysis of macroscopic ionic currents and kinetic simulations have led to predictions that the hERG activation pathway involves a voltage-independent, rate-limiting step. Characterization of the voltage-dependence of the activation time constant $\left(\tau_{\text {act }}\right)$ showed that hERG channels activate in a sigmoidal fashion (Trudeau et al., 1995; Liu et al., 1996; Wang et al., 1997), which suggests that the activation pathway is a multi-step process, similar to other Kv channels, such as Shaker (Zagotta et al., 1994a; Fedida and Hesketh, 2001). Detailed analysis of the late phase of activation (sometimes measured as the last $50 \%$ of the activation time course) suggested that $\tau_{\text {act }}$ nears saturation at strongly depolarized potentials $(15 \mathrm{~ms}$ at $+160 \mathrm{mV})$, which is indicative of a voltage-independent step that becomes rate-limiting at positive potentials (Liu et al., 1996; Wang et al., 1997; Subbiah et al., 2004). The strong voltage-dependence of the deactivation rate implies that the final closed to open (and open to closed) transition must be voltage-dependent, thereby requiring that the voltageindependent transition precede the final opening transition (Liu et al., 1996; Wang et al., 1997). Thus, the final linear kinetic model (Figure 3A) for hERG gating proposed by Wang et al. (1997) has three closed states preceding the final open state with a voltageindependent transition between the second and third closed states, immediately before the final opening step. Several other gating schemes have also been proposed, with the major variations having only two closed states (Oehmen et al., 2002) or with the addition of a direct transition from the final closed state to the inactivated state (i.e., channel opening is not required for inactivation; Clancy and Rudy, 2001; Mazhari et al., 2001). All of these models retain a voltage-independent transition prior to the final opening step. A recent comparison of the above kinetic models found that the simple linear model first proposed by Wang et al. (1997) is best able to reproduce the qualitative and quantitative behavior of hERG channels under voltage-clamp and action potential simulations (Bett et al., 2011).

The model for hERG gating proposed by Wang et al. (1997) is limited in that it does not correlate transitions in the model with changes in individual $\alpha$-subunits, in contrast to gating schemes such as the Zagotta Hoshi Aldrich (ZHA; Zagotta et al., 1994a) 


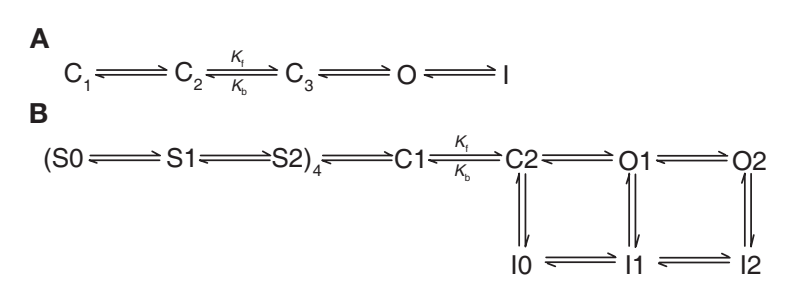

FIGURE 3 | Proposed schemes to describe hERG channel gating.

(A) the three closed state model proposed by Wang et al. (1997). Kf and Kb represent voltage independent transitions that are rate limiting for opening at strongly depolarized potentials. (B) The modified gating scheme proposed by Piper et al. (2003) to account for the biphasic nature of hERG gating currents and multiple sequential voltage-dependent transitions of the voltage sensor

and Schoppa and Sigworth (S-S; Schoppa and Sigworth, 1998a) models for Shaker channel gating that were based on combined gating current, macroscopic ionic current and single channel current data. For example, the S-S model suggests that each subunit independently proceeds through three voltage-dependent gating steps, followed by two concerted transitions to a final closed state and then to the open state (Schoppa and Sigworth, 1998a; Fedida and Hesketh, 2001). Following the first records of hERG gating currents, Piper et al. (2003) proposed an updated Markov model that incorporated the Wang et al. (1997) hERG model as well as the ZHA and S-S Shaker models (Figure 3B). This model takes into account the biphasic nature of the hERG gating currents and, similar to the ZHA and S-S models, assumes that each subunit independently undergoes two sequential, voltage-dependent transitions before the channel proceeds in a concerted fashion through two sequential closed states from where the channel may then open. Also like the S-S model, the early independent transitions were thought to correspond to movement of gating charge and were rate-limiting at lower membrane potentials. The voltageindependent transition between the last two closed states that is rate-limiting at strongly depolarized potentials is maintained, although the rate of the reverse transition is several-fold higher than in the original Wang et al. model. The expanded model presented by Piper et al. (2003) is reasonably able to simulate ionic and gating currents of WT and inactivation-deficient mutant hERG channels under voltage-clamp conditions, although its ability to accurately predict currents under action potential simulation has not been assessed.

\section{S4 MOVEMENT AND GATING ARE REGULATED BY INTRA-SUBUNIT INTERACTIONS}

The sections above have focused primarily on the location and motion of positively charged residues in the S4 segment of the voltage-sensing domain. We now turn attention toward the potential interactions between the S4 segment and the three other transmembrane segments that make up the voltage-sensing domain (i.e., S1, S2, and S3), which may contribute to the regulation of voltage-dependent S4 movement.

Early charge neutralization studies of the S4 segment in Shaker channels, showed that two S4 mutants, K5Q (K374Q) and R6Q
(R377Q), did not produce functional channels (Papazian et al., 1991). Expression and function of K5Q, and to a lesser extent $\mathrm{R} 6 \mathrm{Q}$, could be rescued when paired with the neutralization of highly conserved negative charges at the intracellular ends of S2 (E293Q) or S3 (D316N), which suggests that interactions between these charged residues are important for protein folding and maturation (Papazian et al., 1995). Using a similar charge reversal approach (i.e., K5E, E293K, D316K), Tiwari-Woodruff et al. (1997, 2000) suggested that specific interactions occur between K5, E293, and D316 in the same subunit and that these interactions may be electrostatic in nature (but see below). Potential electrostatic interactions were also shown between E283 at the extracellular end of S2 and R3 and R4 in S4 (Tiwari-Woodruff et al., 1997, 2000). As E283 did not exhibit state-dependent accessibility to external MTS reagents (Wang et al., 1999; Tiwari-Woodruff et al., 2000), it likely does not move relative to the electric field during channel activation. Thus, this latter result suggests that E283 interacts sequentially with the charge-carrying R3 and R4 residues as they move through the electric field (Tiwari-Woodruff et al., 2000). The stabilization of partially activated and/or open states of the channel by interactions of E283 with R3 and R4 is further supported by the observation that disrupting these interactions, by mutation of E283, caused a depolarizing shift in the $G-V$ relationship (Papazian et al., 1995). In sum, the above findings, in combination with previous data on gating charge movement and accessibility are consistent with the hypothesis that R3 and R4 interact with E283 near the extracellular surface of the channel during activation, while interactions at the intracellular face of the channel between E293, K5, and D316 tend to stabilize the closed state. More recently, experiments with neutral unnatural amino acid homologs for E283, E293, and D316 have suggested that, while the interactions involving E283 are indeed electrostatic in nature, E293 and D316 do not participate in electrostatic interactions with S4 and are instead important in maintaining the water-filled crevice at the base of the voltage-sensing domain (Pless et al., 2011). Mutation of E293, D316, and/or K5 may thus change the local dielectric within the water-filled vestibule, which could result in an altered electric field and the observed effects of these mutations on voltage-dependent Shaker gating. Determination of the X-ray crystal structures for Kv1.2, the Kv1.2-Kv2.1 paddle chimera and KvAP in the activated conformation confirm the close proximities of E283 to R4 and of K5 to E293 and D316 (Jiang et al., 2003; Long et al., 2005a, 2007; Chen et al., 2010).

Compared to the interactions occurring in the voltage-sensing domain in the activated conformation, for which there is high resolution structural information, the nature of the interactions occurring in the resting closed state are less clear. The ability of R1 and R4 Shaker mutants to conduct protons or monovalent cations through the voltage-sensing domain at hyperpolarized and depolarized potentials, respectively (Starace and Bezanilla, 2004; Tombola et al., 2005), suggests that these residues occupy similar positions in the resting and activated states. Mutation of E283 modulated these "gating pore" currents, suggesting that, like R4, R1 approaches E283 during gating (Tombola et al., 2005). Disulfide bond formation between R1C and I241C in S1 or I287C in S2 in the closed state (Campos et al., 2007) also supports close proximity of R1 and E283 in the resting state. Several structural models 
of Shaker, Kv1.2 and KvAP channels in the resting state have been developed using these experimental data to constrain the position of R1 close to E283 (Yarov-Yarovoy et al., 2006; Campos et al., 2007; Pathak et al., 2007; Nishizawa and Nishizawa, 2008; Schow et al., 2010; Vargas et al., 2011). Using molecular dynamics simulations to calculate the gating charge transferred when the Kv1.2 S4 segment moves from a resting position, where R1 and E283 interact, to an activated conformation, where $\mathrm{R} 4$ and $\mathrm{E} 283$ interact, resulted in a value of $\sim 12-12.7 \mathrm{e}_{0}$ (Khalili-Araghi et al., 2010), which is in good agreement with experimental findings (Bezanilla, 2000).

In contrast to the data discussed above, other experimental evidence suggests that Shaker R1 does not interact with E283 at rest and is instead located in a "gating charge transfer center" formed by F290 further down in the S2 segment, in the intracellularly accessible water-filled crevice defined by E293 in S2 and D316 in S3 (Figure 4A; Tao et al., 2010; Lin et al., 2011). In the Kv1.2 and Kv1.2-Kv2.1 chimera crystal structures the highly conserved F233 residue (analogous to Shaker F290) forms part of the $10 \AA$ thick hydrophobic layer in the voltage-sensing domain that focuses the transmembrane electric field and also separates the intracellular and extracellularly accessible water-filled crevices in the voltagesensing domain. Based on this, F233 has been described as a "hydrophobic plug" and "Phe gap" (Long et al., 2007; Chen et al., 2010). The crystal structures, which depict S4 in its activated conformation, show that F233 separates K5 (i.e., Shaker K374) from the extracellular crevice and it was proposed that the S4 charges move across the electric field by sequentially flipping past the phenylalanine residue, such that $\mathrm{R} 1$ occupies this position at rest, consistent with a translocation of the S4 segment of $10 \AA$ (Long et al., 2007; Chen et al., 2010; Tao et al., 2010). It was recently shown that the F290W mutation in Shaker increased the affinity of the gating charge transfer center for lysine residues, presumably due to an induced cation $-\pi$ relationship (Tao et al., 2010; Pless et al., 2011). In support of a resting R1-F290 interaction, the charge-conserving R1K mutation (in the F290W background) resulted in a right-shifted G-V relationship indicative of a stabilized resting conformation and increased interaction between the substituted $\mathrm{K} 1$ charge and the tryptophan residue at 290 (Tao et al., 2010). A recent assessment of the ability of $\mathrm{I} 287 \mathrm{H}$ and $\mathrm{R} 1 \mathrm{H}$ in Shaker to form a binding site for extracellular $\mathrm{Zn}^{2+}$ has also shown that, at $-80 \mathrm{mV}$, only a fraction of channels have voltage sensors where $\mathrm{I} 287 \mathrm{H}$ and $\mathrm{R} 1 \mathrm{H}$ are in close enough proximity to bind $\mathrm{Zn}^{2+}$ and this proportion decreases with changes in potential in either direction (Lin et al., 2011). This is consistent with voltage-dependent translocation of S4 that moves R1 further away from I287, and supports the idea of an inward movement of R1 away from $\mathrm{I} 287$ and, thus, E283 upon hyperpolarization. $\mathrm{I} 287 \mathrm{H}$ can form a $\mathrm{Zn}^{2+}$ binding site when paired with $\mathrm{A} 359 \mathrm{H}$ in the S3-S4 linker and $\mathrm{Zn}^{2+}$ binding to this site stabilizes the resting state (Lin et al., 2011). These findings lend strong support to the hypothesis that R1 does not interact with E283 in the fully closed state, but is instead located further toward the intracellular space, perhaps in the vicinity of the gating charge transfer center. It is possible to reconcile these data with previous studies if one considers that interactions with the gating charge transfer center do not preclude R1 from approaching I287 and interacting with E283 during transitions through partially activated states.

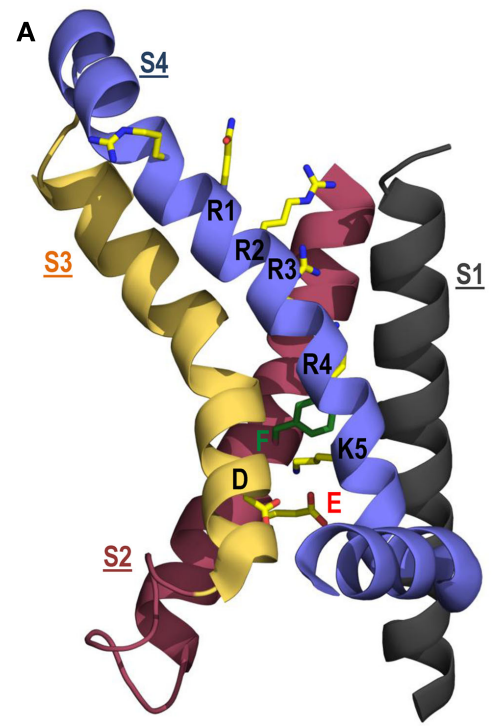

FIGURE 4 | Structural models of the voltage-sensing domain and pore domain. (A) ribbon representation of the voltage-sensing domain (S1, black: S2, red; S3, yellow; S4, blue) in an open conformation based on the Kv1.2-Kv2.1 chimera crystal structure (Long et al., 2007). Side chains found in the proposed gating charge transfer center (D316, E283, and F290) and along S4 (R1, R2, R3, R4, K5) are colored according to atom type: C, yellow; N, blue; $\mathrm{O}$, red; phenylalanine, green. For clarity, the S1-S2 linker has been omitted.

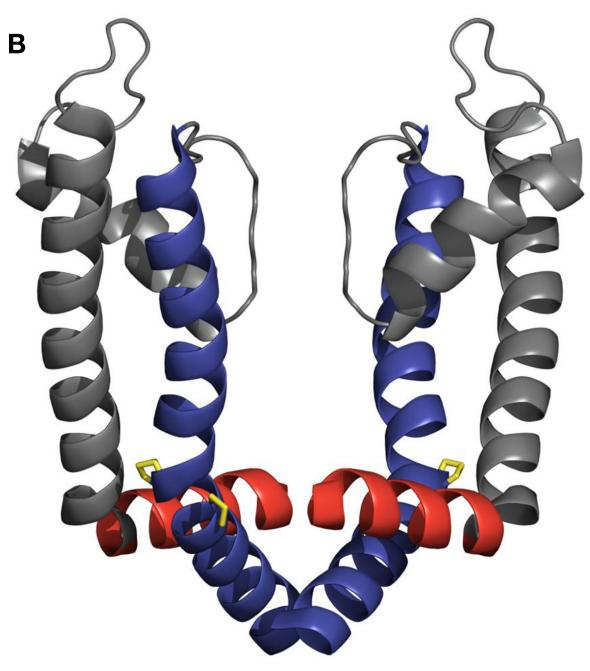

(B) Ribbon representation of the open pore domain (S5, gray; S6, blue) and S4-S5 linker (red), based on the Kv1.2-Kv2.1 chimera crystal structure, showing the close apposition of the S4-S5 linker and distal S6 helices. Proline residues that form the highly conserved PxP motif in the distal S6 helix and allow electromechanical coupling via the S4-S5 linker are highlighted in yellow. Only two subunits of the tetramer are shown (two subunits have been removed for clarity). 
As discussed above, the $\mathrm{S} 4$ segment of hERG contains a lysine residue at the extracellular (K1; K525) and intracellular extremes (K6; K538), while Shaker-type channels have an arginine at the top of S4 (Figure 1C). The presence of both K1and K6 have been reported to stabilize the closed state of hERG: mutations at these positions caused a hyperpolarizing shift of the $G-V$ relationship and accelerated activation (Liu et al., 2003; Subbiah et al., 2004, 2005; Zhang et al., 2004, 2005; Piper et al., 2005; Subbotina et al., 2010). Given that substitution of K1 or K6 with neutral (Q, C, $\mathrm{W}$, or $\mathrm{A}$ ) and/or charged ( $\mathrm{D}$ or $\mathrm{E}$ ) residues caused destabilization of the closed state, the lysine interactions are unlikely to be electrostatic in nature. The stabilization of the hERG closed state by $\mathrm{K} 6$ at the bottom of S4 is in stark contrast to the proposed role played by $\mathrm{K} 5$ at the bottom of the Shaker S4 in stabilizing the open state (see above, Tao et al., 2010; Lacroix and Bezanilla, 2011; Pless et al., 2011). Importantly, K6 is one of two consecutive positively charged residues at the bottom of the hERG S4 (R5 or R537 being the other) and either could potentially act as the analogous residue to Shaker K5. Although not yet formally assessed, the proximity of these two consecutive basic residues may contribute to the differential effects of substituting $\mathrm{K} 6$ in hERG, compared to K5 in Shaker. In contrast to the closed-state stabilization of $\mathrm{K} 1$ and $\mathrm{K} 6$, neutralization or charge reversal of the hERG S4 arginines (R2, R3, R4, R5) generally caused a moderate depolarizing shift of the $G-V$ relationship (Subbiah et al., 2004, 2005; Zhang et al., 2004, 2005; Piper et al., 2005). Intriguingly, the arginine mutants often also affected the time constants for channel activation and deactivation (Subbiah et al., 2004, 2005; Zhang et al., 2005). Together, these results suggest that the arginines in the middle of S4 may be involved in interactions that stabilize the open state or, alternatively, in facilitating transitions leading to the open state. Overall, R2 and R3 were shown to have the largest effects on both the voltage-dependence and rate of channel opening (Subbiah et al., 2004, 2005; Piper et al., 2008). As the analogous residues in Kv1.2 have been suggested to form salt-bridges within the activated conformation of the voltage-sensing domain seen in the Kv1.2 crystal structure (Long et al., 2005a), these observations have been considered to suggest that interactions involving R2 and R3 are particularly important in stabilizing partially activated states and the slow activation of hERG (Piper et al., 2005; Subbiah et al., 2005). Similar parallels can also be drawn with the roles of Shaker R3 and R4 in stabilization of partially activated conformations (see above).

As with Shaker channels, the potential interaction partners for the S4 basic residues in hERG channels have been studied, albeit less extensively. The voltage-sensing domains of hERG and other eag family members possess three conserved negative charges in S2 (D456, D466) and S3 (D501), as well as three additional acidic residues not conserved across other $\mathrm{Kv}$ channel families: D411 (S1), D460 (S2) and D509 (S3), and an extra lysine at the base of S1 (K407; Figure 1A,C). It is conceivable that the presence of these non-conserved negative charges in hERG may result in additional electrostatic interactions with $\mathrm{S} 4$ basic residues and perhaps contribute to the uniquely slow gating of hERG channels. Using double mutant cycle analysis and charge reversal mutations, Zhang et al. (2005) found evidence for interactions between
K1 and D456 (equivalent to the proposed R1-E283 interaction in Shaker) and between K6 and D411 at the base of S1, both of which were proposed to stabilize the closed state and contribute to slow hERG activation. The latter interaction would be absent in Shaker channels, which do not possess a D411 equivalent in S1. The same study showed that D456 also interacts with R2 during partially activated states and that this interaction facilitates activation. The same approach was used more recently with charge neutralization mutants to show that R3 is energetically coupled during activation to all of the acidic residues in the voltage-sensing domain (Piper et al., 2008), although note that a R3-D411 interaction is inconsistent with the results of Zhang et al. (2005), which specifically showed that R3 did not interact with D411. The sum of these findings is a picture of hERG activation that involves a voltage sensor that is stabilized at the top and bottom by interactions involving $\mathrm{K} 525$ and $\mathrm{K} 538$, respectively. Depolarization would provide sufficient energy to break these interactions and new interactions of R528 and R531 with D456 would form as the S4 segment is extruded. Based on findings in closely related eag channels, R534 also likely interacts with D456 in the activated state (Silverman et al., 2003), consistent with the salt-bridges observed between the analogous residues in the Kv1.2 crystal structure (Long et al., 2005a). Support for an R534-D546 interaction is also provided by the observation that $\mathrm{Mg}^{2+}$ binding to the pocket of negative charges in hERG formed by D456 and the non-conserved D460 and D501 residues accelerated deactivation, presumably by disrupting the ability of R534 to interact with D456 and, thus, destabilizing the activated state (Lin and Papazian, 2007). Thus, the sequential coupling of the S4 arginines to D456 at the top of S2 during hERG activation is very similar to the model for Shaker channel activation proposed by Papazian and colleagues (see above). This is in good agreement with the observation that the extent of Shaker and hERG S4 extrusion appears to be quite similar (as discussed above, Elliott et al., 2009).

\section{IS THE GATING CHARGE TRANSFER CENTER DESCRIBED FOR SHAKER CONSERVED IN hERG?}

Recently there has been considerable renewed interest in the regulation of the movement of S4 charges across the electric field in response to changes in voltage. In large part, interest has focused on the potential role of a highly conserved phenylalanine residue in S2 (F290) that forms part of a hydrophobic plug at the base of the voltage-sensing domain (Chen et al., 2010). As mentioned above, it was recently suggested that F290 acts as a gating charge transfer center in Shaker channels (Tao et al., 2010). Introduction of a tryptophan residue at position 290 resulted in preferential interaction of the gating charge transfer center with lysine (over arginine residues), such that the closed or open conformations of the voltage sensor could be stabilized depending on whether a lysine occupied the first or fifth charged position in S4, respectively (Tao et al., 2010). Although a cation $-\pi$ interaction between lysines and the native phenylalanine residue was ruled out (Tao et al., 2010; Lacroix and Bezanilla, 2011), the increased affinity for lysine caused by the F290W mutation is likely due to a preferential cation $-\pi$ interaction with the indole ring of the substituted tryptophan residue (Pless et al., 2011). Currently, there 
is still debate over whether F290 regulates the movement of each individual S4 gating charge across the electric field in WT Shaker (Tao et al., 2010) or if this interaction is limited to only the fourth arginine (R4; Lacroix and Bezanilla, 2011). Nevertheless, it seems clear that in the F290W mutant, the open state of the channel is stabilized and activation is rapid with an arginine at the top of S4 and a lysine at the bottom (i.e., R1, K5; Tao et al., 2010).

To date, there is little published information regarding the importance of the gating charge transfer center in voltage-gated ion channels other than Shaker. The phenylalanine residue in S2 thought to form the gating charge transfer center in Shaker (F290) is conserved in hERG channels (F463; see Figure 1C). We have examined the effects of charge-conserving mutations in the S4 segments of hERG channels to address the question of whether a gating charge transfer center also regulates S4 movement and channel activation in hERG channels. In Shaker, switching the position of the arginine at the top of S4 and the lysine at the bottom (i.e., R1K-K5R) in the F290W background caused a dramatic slowing of channel activation, deactivation, and gating charge movement, consistent with the stabilization of the closed state by the presence of a lysine at the top, but not the bottom, of S4 (Tao et al., 2010). Intriguingly, WT hERG channels, which present slow activation, deactivation, and gating charge movement, have a lysine (K1) as the outermost $\mathrm{S} 4$ charge and the arginine residue immediately adjacent to K6, R5, aligns better with the repeating sequence of S4 charges (i.e., $\mathrm{KxxRxxRxxR}^{537} \mathrm{~K}^{538}$ ) and, thus, with Shaker K5 (see Figure 1C). We have asked whether the different nature of the charged residues at the ends of the S4 helix (i.e., lysine vs. arginine) contribute to the unique voltage-dependent gating properties of hERG channels. We found that the chargeconserving K1R mutation in hERG causes a large left-shift of the $G-V$ relationship (Cheng et al., 2012), consistent with previous reports that hERG $\mathrm{K} 1$ is important in stabilization of the closed state of the channel (Zhang et al., 2004, 2005; Subbiah et al., 2005). This finding is also in agreement with the observation in Shaker channels that a lysine at the outer end of the S4 segment imparts greater resting-state stability than an arginine residue (Tao et al., 2010) and is therefore supportive of a gating charge transfer center mechanism. Investigating interactions at the bottom of S4, we found that both R5K and K6R mutations exhibited a right-shifted voltage-dependence of activation (Cheng et al., 2012). These data suggest that a lysine at position 5 (i.e., R5K) destabilizes the open state, but at position 6 a lysine stabilizes the open state. Although the latter is in line with the finding in Shaker that a lysine residue at the bottom of S4 stabilizes the open state, the finding that a lysine at position 5 destabilizes the open state is in contrast to the results in Shaker (Tao et al., 2010). These data raise the question as to which residue, $\mathrm{R} 5$ or K6, occupies the gating charge transfer center in the activated state. Furthermore, it is apparent that the putative interactions between the proposed gating charge transfer center and the charged residues at the base of S4 in hERG appear to be more complex than those described in Shaker channels. The different identities (i.e., lysine or arginine) of the charged residues at the extreme ends of the S4 segments in hERG and Shaker channels and their resulting interactions with the putative gating charge transfer center may contribute to the characteristically slow gating of hERG channels.

\section{ROLE OF THE S4-S5 LINKER IN hERG AND SHAKER CHANNEL GATING}

\section{THE S4-S5 LINKER COUPLES THE VOLTAGE-SENSING AND PORE DOMAINS}

As mentioned above, the S4-S5 linker plays a pivotal role in $\mathrm{Kv}$ channel gating. This short connecting linker lies at the internal lipid interface and couples translation of the S4 voltage sensor segment to opening and closing of the intracellular pore gate. Crystal structure representations of the Kv1.2 channel open state show that the S4-S5 linker lies in close apposition to the distal S6 segment of the same subunit (Figure 4B) and have led to the proposal that the S4-S5 linker electromechanically couples voltage sensing with channel opening (Long et al., 2005a; Chen et al., 2010). This critical role of the S4-S5 linker in Kv channels is also supported by functional evidence. Chimeric constructs of Shaker and KcsA channels demonstrated that voltage dependent gating requires the distal S4-S5 linker of Shaker from L385 to L399 (Lu et al., 2002). Replacement of the Shaker S4-S5 linker, along with a portion of the distal S6 helix, with the corresponding sequence from the voltage insensitive KcsA channel specifically abolished voltage dependent gating in Shaker channels. A similar chimeric approach using voltage-gated Kv1.5 and Kv2.1 channels showed that voltage-dependent gating required specific contacts between the S4 and S5 linker with the distal S6 helix, which allow for mechanical translation of voltage sensor movement to the S6 pore gate (Labro et al., 2008). More recently, gating current and VCF measurements of voltage sensor dynamics in Shaker channels showed that direct coupling between R394, E395, and L398 in the S4-S5 linker and Y485 in the distal S6 segment stabilizes the open state of channels (Batulan et al., 2010). A similar coupling role for the S4S5 linker has been reported in hERG channels. Charge reversal of D540 in the S4-S5 linker destabilized the closed state of hERG causing channels to re-open upon hyperpolarization (Sanguinetti and $\mathrm{Xu}, 1999$ ). Alanine substitution of R665, but not other neighboring residues, in the distal S6 segment prevented re-opening in D540K channels, suggesting that electrostatic repulsion between D540K and R665 causes re-opening of the intracellular activation gate at hyperpolarized potentials (Tristani-Firouzi et al., 2002). Subsequent studies showed that cross-linking of cysteine residues engineered at D540 in the S4-S5 linker and L666 in the distal S6 segment stabilized the closed conformation of the hERG activation gate (Ferrer et al., 2006). Interestingly, cross-linking of these residues also immobilized a portion of voltage sensor gating charge, consistent with a role of the S4-S5 linker in electromechanical coupling in hERG channels.

Outward movement of the S4 helices is translated, via the S4S5 linkers, into a pivoted bending of the lower portion of the S6 helices that opens the aperture of an intracellular gate to permit ion permeation. Comparison of channel structures crystallized in the open state, such as Kv1.2, KvAP, and MthK (Jiang et al., 2002b, 2003; Long et al., 2005a; Chen et al., 2010), with those in the closed state, such as KcsA, (Doyle et al., 1998) suggest that the intracellular 
gate in Kv channels is formed by the convergence of the S6 helices near a highly conserved PxP motif that introduces a kink in the $\alpha$-helical structure to allow for electromechanical coupling with the S4-S5 linker (Figure 4B). Upon depolarization, movement of the S4-S5 linker is predicted to cause radial displacement of the lower S6 helices in a manner that is dependent on the flexibility of a conserved "glycine hinge" higher up in S6. This model of activation is supported by substituted cysteine accessibility studies showing that residues below the activation gate are accessible to chemical modification by MTS reagents in both closed and open states, while residues above are only accessible in the open state (Liu et al., 1997). Furthermore, sites above the activation gate were protected from MTS modification by binding of pore blockers, such as tetrabutylammonium (TBA), tetraethylammonium (TEA), and the N-terminal inactivation particle (Liu et al., 1997; del Camino et al., 2000). A scanning mutagenesis study pinpointed V478 and F481 as sites that occlude ion permeation in the closed state (Hackos et al., 2002), the former of which has been described as a hydrophobic seal that prevents $\mathrm{K}^{+}$access to the inner cavity of the channel (Kitaguchi et al., 2004). This putative hydrophobic seal in Shaker lies approximately one helical turn below the PVP motifinduced kink in S6 and two turns above the S4-S5 linker contact point in S6 (Y485) described by Batulan et al. (2010). Based on these structural and functional data, a mechanism by which S4-S5 linker coupling of voltage sensor movement displaces the distal S6 helices to dilate an intracellular pore aperture around V478 (in Shaker) presents a compelling model to describe $\mathrm{Kv}$ channel activation gating.

\section{A MISSING PXP MOTIF IN hERG MAY CONTRIBUTE TO ITS UNIQUE GATING}

Interestingly, hERG channels lack the well conserved PxP motif in S6. This has raised questions as to the location of the intracellular gate in hERG channels and how it is coupled to S4 movement. Introduction of the PVP motif into hERG channels (substituting the $I^{655} \mathrm{~F}^{656} \mathrm{G}^{657}$ triplet) apparently locked channels in the open state (Fernandez et al., 2004). This suggests that the structure of the hERG activation gate and its coupling to the voltage-sensing unit is different from that in Kv channels such as Shaker. Robertson and co-workers performed a cysteine scan of the distal portion of S6 in hERG in an attempt to determine the structural elements that define the intracellular pore gate (Wynia-Smith et al., 2008). Cysteine substitution at Q664, Y667, and S668 induced slow deactivation and constitutive channel opening at negative potentials. Mapping these sites onto models of the open and closed states of the channel (based on Kv1.2 and MlotiK1 crystal structures, respectively) suggested that they form a gating ring that occludes the permeation pathway in the closed state. Interestingly, this occlusion site is positioned in the vicinity of the S4-S5 linker contact point in S6 (L666) described by Ferrer et al. (2006) and approximately two helical turns below the location of the gate (V478) in Shaker channels. This suggests that the position of the pore gate in hERG channels may be different from that described in Shaker. If this is the case, the mechanism by which S4-S5 linker interactions with S6 drive opening and closing of the gate is an important issue that will require further investigation in order to fully understand hERG's unusual gating characteristics.

\section{CRITICAL STRUCTURAL FEATURES OF THE hERG AND SHAKER S4-S5 LINKERS}

Analysis of the Kv1.2 channel structure in the open configuration shows that the S4-S5 linker spans residues S311-A323 and forms three turns of an amphipathic helix (Long et al., 2005a). Similar findings were reported from NMR structures of the Shaker channel S4-S5 linker isolated and incorporated into lipid micelles (Ohlenschlager et al., 2002). The amphipathic nature of the helix is consistent with a position approximately parallel to the plane of the membrane, and suggests that the short helix lies at the interface of the internal phospholipid layer. NMR structures have also been reported for the S4-S5 linker of hERG channels (Gayen et al., 2012; Ng et al., 2012). These structures of isolated S4-S5 linker peptide fragments formed in the presence of dodecylphosphocholine (DPC) phospholipid demonstrate that the linker forms a $3_{10}$-helix from S543 to Y545 (Gayen et al., 2012) or an amphipathic $\alpha$-helix from L539 to L550 ( $\mathrm{Ng}$ et al., 2012). The structural discrepancy may simply result from determination of structures using peptide fragments of different length; the S4-S5 linker fragment used by Gayen et al. was shorter (L539-A548) than that used by $\mathrm{Ng}$ et al. (L532-F551). Indeed, residues 539-541 appear to adopt a near helical configuration in the structure presented by Gayen et al. However, as $\mathrm{Ng}$ et al. discuss, this discrepancy may also reflect the dynamic nature of the S4-S5 linker structure. Both studies are somewhat limited in this regard, in that the linker structure is analyzed in an isolated peptide removed from both S4 and S5, which tether the linker at either end, and also from steric interactions with the S6 helices. Despite this limitation, these structures represent the best available structural representations of the S4-S5 linker in hERG channels to date.

In Shaker channels a highly conserved leucine heptad repeat runs from the distal S4 to the proximal S5, spanning the S4-S5 linker. Leucine zippers are important structural motifs that function as dimerization domains for protein-protein and proteinDNA interactions. The role of the putative leucine zipper motif in Shaker channels is unclear, but disruption of the hydrophobic domain sequence was shown to destabilize the open state of the channel (McCormack et al., 1991). In particular, mutation of the $2^{\text {nd }}$ leucine residue $(\mathrm{L} 382 \mathrm{~V})$ within the heptad repeat shifted activation gating of Shaker channels by $\sim+70 \mathrm{mV}$ and reduced the voltage-sensitivity. Gating current analysis in this "V2" mutant showed no change in the amount of charge during activation, but found that the on-gating charge movement became separated into two components. In the V2 mutant, most of the charge moved at negative potentials with a voltage dependence similar to that in WT channels, but a small component of charge moved only at more strongly depolarized potentials that correlates with the voltage dependence of opening of the mutant channels (Schoppa et al., 1992). Subsequent kinetic analysis supported the generation of a model in which the V2 mutation destabilized the open state of the channel by disrupting late cooperative gating transitions without greatly altering voltage sensor transitions earlier in the activation pathway (Schoppa and Sigworth, 1998a,b). Consistent with this idea, 4-aminopyridine, a drug shown to specifically inhibit late gating transitions associated with final concerted channel opening rearrangements, was shown to act on the same component of charge movement altered by the V2 mutation (McCormack 
et al., 1994). Taken together, these data suggest a critical role of the hydrophobic leucine heptad repeat residues within the S4-S5 linker in coupling voltage sensor transitions to the opening of the intracellular pore gate.

Alignment of the S4, S4-S5, and S5 linker regions in Shaker and hERG suggests that an isoleucine/leucine heptad repeat may exist in hERG as in Shaker, but that the repeat is naturally disrupted by the presence of a glycine (G546) at the $2^{\text {nd }}$ leucine position. Given that substitution of the $2^{\text {nd }}$ heptad leucine in Shaker (by the V2 mutation) destabilized the open state of the channel, we have proposed that the presence of a glycine at position 546 may contribute to the slow gating kinetics that are peculiar to the hERG channel (Van Slyke et al., 2010). We showed that restoration of the leucine heptad (by the mutation G546L) destabilized the closed state relative to the open state and accelerated channel activation kinetics. VCF measurements suggested that this was due to faster voltage sensor movement, from which it was inferred that the S4-S5 linker glycine residue may constrain voltage sensor transitions. Interestingly, when G546 was substituted by a number of other different amino acids, the effects on gating were very similar to those of the G546L mutation suggesting that the native glycine residue contributed an additional energy barrier to activation of $1.9-4.3 \mathrm{kcal} / \mathrm{mol}$ (the energy equivalent to $1-2 \mathrm{H}$-bonds). These data suggested that the helical content of the S4-S5 linker is critical to the relative stability of the open and closed states in hERG channels, rather than the presence of a leucine heptad repeat. A "stiffer" helix produced by substitutions of G546 with helix-forming residues, or perhaps by interaction of cytoplasmic domains, biases the channel toward the open state, destabilizing the closed state.

Resolvable helical NMR structures of the S4-S5 linker in Shaker and hERG channels could only be detected in the presence of DPC (Ohlenschlager et al., 2002; Gayen et al., 2012; Ng et al., 2012). It is therefore important to consider whether the functional consequences of S4-S5 linker mutations, such as those of G546, are due to altered interactions between the side-chain at the position of interest and the surrounding lipid environment. Interestingly, although the S4-S5 linker is an amphipathic helix, both NMR structures of the S4-S5 linker of hERG channels place Y542 and G546 on the same face of the helix (Gayen et al., 2012; Ng et al., 2012). Both of these sites have been implicated in interactions of the S4-S5 linker with the N-terminus. Y542C was shown to cross-link with V3C in the N-terminal domain (de la Peña et al., 2011) and $N$-ethylmaleimide-labeled G546C prevented access of the N-terminus to its interaction site (Wang et al., 1998). This functional evidence suggests that although these residues align along the hydrophobic surface of the helix in the NMR structures, this face may be exposed to solvent in the full-length channel. Further evidence against lipid interaction altering effects of G546 mutations is that the G546A mutation (which does not significantly alter the side chain structure nor, therefore, lipid interactions) had just as dramatic an effect on gating as the introduction of bulky hydrophobic residues that would be expected to alter lipid interactions dramatically. In its native configuration within the channel, the extent to which the S4-S5 linker interacts with the surrounding lipid remains to be determined. The short S4-S5 linker, tethered at either end by S4 and S5 and crossing over and interacting with S6 clearly resides, at least partially, within a proteinaceous environment forming multiple protein-protein interactions. Furthermore, the presence of internally accessible canaliculi that have been proposed to project along the internal length of S4 (Bezanilla, 2000), confer additional complexity when considering the manner in which the channel protein interacts with its lipid environment at this critical location. A recent LRET study of the structural dynamics of the S4-S5 linker in response to voltage sensor gating in KvAP channels showed that the linker is highly dynamic, experiencing both translational and rotational motions (Faure et al., 2012). Such dynamics are consistent with the linker's role in electromechanical coupling, and will need to be taken into account when evaluating its involvement in potential protein-lipid interactions.

\section{CYTOPLASMIC DOMAINS MAY INTERACT WITH THE S4-S5 LINKER TO MODULATE GATING}

Deletion of the majority of the N-terminus, from residues 2354 or 2-373, dramatically accelerated hERG channel deactivation (Schonherr and Heinemann, 1996; Spector et al., 1996). Subsequent studies showed that residues 2-16 were responsible for regulating the rate of deactivation (Wang et al., 1998) and that slow deactivation could be restored in N-terminal deleted channels by the application of an $\mathrm{N}$-terminal peptide corresponding to this sequence (Wang et al., 2000) or to residues 1-135 (Morais Cabral et al., 1998). These data demonstrated that the distal N-terminus stabilizes the open state of the hERG channel. A high resolution crystal structure of the N-terminus of hERG from residue 26135 was solved by Morais Cabral et al. (1998). Onto this, the authors mapped the functional effects of individual mutations, which demonstrated that residues F29 and Y43 had a particularly large effect on deactivation gating and showed that these residues were located within a hydrophobic patch that may form a complex interaction surface. Recently, the NMR structure of the distal $\mathrm{N}$-terminus was reported (Li et al., 2010; Muskett et al., 2011; Ng et al., 2011). This structure contains residues 1-135 and therefore includes the first 25 amino acids missing from the previous crystal structure. Driven by the NMR structure of the distal portion of the N-terminus, along with mutational analysis, Vandenberg and co-workers have suggested that residues R5 and G6 are essential for slow deactivation of hERG gating and that the remainder of the $\mathrm{N}$-terminus contributes to deactivation gating by orienting the distal N-terminal tail in such a manner as to promote interaction with the core of the channel (Ng et al., 2011).

Interestingly, deletion of regions of the proximal N-terminus (residues 138-373) have the opposite effect to distal deletions, shifting the voltage dependence of activation in the hyperpolarizing direction (Viloria et al., 2000; Gómez-Varela et al., 2002) and accelerating the time course of activation (Alonso-Ron et al., 2008), suggesting that this region is important in stabilizing the closed state of the channel. This is supported by the observation that a cluster of charged residues (three positive and one negative between residues 362 and 372) in the proximal $\mathrm{N}$ terminus strongly stabilized the closed state of the channel (Saenen et al., 2006). Thus, it is clear that the $\mathrm{N}$-terminus plays a key role in dictating the unusual activation and deactivation gating of hERG channels. 
hERG channel deactivation is also modified by deletions of the C-terminus. Deactivation was markedly accelerated following deletion of the distal 236 residues of the C-terminus and was not further accelerated by deletion of the $\mathrm{N}$-terminal 2-354 residues (Aydar and Palmer, 2001). These data suggest that the C- and Ntermini act in concert to modify deactivation behavior of hERG channels. Subsequent studies support such a functional interaction. A strategy of introducing positively charged lysine residues within the C-terminus was used to discover putative functional interaction sites (Al-Owais et al., 2009). This study showed that a number of lysine substitutions within the cyclic nucleotide binding homology domain (cNBHD) accelerated deactivation gating. The authors mapped these sites onto a homology model of the cNBHD and described a banded pattern of distribution suggesting a broad interaction surface similar to that identified in the N-terminus (Morais Cabral et al., 1998). Recently, the NMR structure of the N-terminal 1-26 residues of hERG channels was mapped onto a structural model of the cNBHD (Muskett et al., 2011). This study highlighted the presence of several negatively charged patches on the surface of the cNBHD that align well with the docked Nterminal structure, which presents positive charges along one face of an amphipathic helix formed by residues 13-23. Charge reversal of a number of these negative acidic residues accelerated deactivation suggesting that charge-charge interactions occur with the $\mathrm{N}$-terminus and a model was generated predicting electrostatic interactions of R4, R5, and $\mathrm{H} 7$ in the $\mathrm{N}$-terminus with D843, E847, and D850 in the cNBHD. Consistent with a functional interaction between $\mathrm{N}$ - and C-termini, application of an N-terminal peptide could not restore slow deactivation in N-terminally truncated channels that lacked the cNBHD (Gustina and Trudeau, 2011). Taken together, these data suggest that the $\mathrm{N}$ - and C-termini of hERG channels interact to modify deactivation behavior. Additional discussion of this topic may also be found in a companion review by Barros et al. (2012).

In Shaker channels mutations within the S4-S5 linker alter interactions with an $\mathrm{N}$-terminal domain that binds within and occludes the intracellular pore conferring rapid N-type inactivation (Isacoff et al., 1991; Holmgren et al., 1996; del Camino et al., 2000). A mutagenic scan of the S4-S5 linker revealed five sites (L385, T388, S392, E395, and L396) that, when mutated, altered N-type inactivation of Shaker channels (Isacoff et al., 1991) and a subsequent study showed that chemical modification of A391C altered binding of a soluble N-terminal inactivation domain peptide (Holmgren et al., 1996). These data suggest that the N-terminal inactivation domain comes into close proximity with the S4-S5 linker in Shaker channels. Similarly, numerous reports have suggested that the hERG $\mathrm{N}$-terminus impacts gating transitions via interactions with the S4-S5 linker. Modification of a cysteine residue substituted at G546 in the S4-S5 linker with $\mathrm{N}$-ethylmaleimide interfered with the action of the N-terminus, suggesting that a bulky adjunct attached to the S4-S5 linker impedes binding of the N-terminus (Wang et al., 1998). Using VCF, we have shown that truncation of the N-terminus $(\Delta 2-354)$ accelerated the fluorescence report of S4 return (Van Slyke et al., 2010), suggesting that the N-terminus directly modifies voltage sensor configurations. Further support for a functional interaction between the S4-S5 linker and the N-terminus comes from a study showing that a cysteine residue introduced at Y542 in the S4S5 linker prevented the restoration of slow deactivation induced by the application of a 1-135 peptide fragment in N-terminal truncated channels (Fernández-Trillo et al., 2011). Moreover, Y542C could cross-link with a cysteine substituted at residue V3 in the N-terminus (de la Peña et al., 2011). This interaction occurred preferentially in the closed state and could be reversed under reducing conditions. This provides strong evidence that the distal N-terminus is within close proximity of the S4-S5 linker, at least in closed channels, and builds upon previous reports from the same research group that a number of S4-S5 linker mutations seem to mimic the acceleration of deactivation observed in $\mathrm{N}$ terminal mutant constructs (Alonso-Ron et al., 2008). Structural evidence also points to a direct interaction of the $\mathrm{N}$-terminus with the S4-S5 linker. Li et al. (2010) presented the NMR structure of amino acids $1-135$ of the N-terminus of hERG and reported chemical shifts of several residues within the $\mathrm{N}$-terminal region after the addition of an S4-S5 linker peptide that included residues R541-V549. Such perturbations were interpreted to indicate a direct interaction between the S4-S5 linker and N-terminus peptides, and residues 86-94 were predicted to be key candidates for binding. This interaction site is different from other $\mathrm{N}$-terminal interaction sites shown from functional studies, which describe key roles for F29 and Y43 (Morais Cabral et al., 1998), V3 (de la Peña et al., 2011), and R5 and G6 (Ng et al., 2011). This suggests that multiple contact points may govern the functional interaction between the $\mathrm{N}$-terminus and the S4-S5 linker. Alternatively, since the S4-S5 linker is integral to the dynamics of electromechanical coupling, S4-S5 linker interactions with the N-terminus may be state-dependent and this may account for some of the differences observed. Additional functional studies by Ng et al. (2012) identified a complex pattern of gating modifying behaviors in channels in which S4-S5 linker residues were mutated. This was interpreted to suggest that the S4-S5 linker not only couples voltage sensor movement to pore opening in hERG channels, but also takes part in complex interactions as the channel transitions between closed and open states. In particular, S543, Y545, G546, and A548 were identified as key residues participating in interactions with other channel domains. This observation is consistent with previous studies that identified G546 as a critical site for hERG gating that is within proximity with the N-terminal domain (Wang et al., 1998; Van Slyke et al., 2010). It is conceivable that these contact points (S543, Y545, G546, and A548) mediate multi-site interactions with a complex formed by the $\mathrm{N}$ - and C-termini that modulate the open state stability of hERG channels. Our VCF data suggest that this may occur via alterations to the relative stability of resting/active voltage sensor configurations (Van Slyke et al., 2010). Furthermore, our evidence that flexibility of the S4-S5 linker may be a key determinant of rate-limiting voltage sensor gating could suggest that such cytoplasmic domain interactions with the S4-S5 linker may alter gating by altering the flexibility of linker. Taken together, this series of structural and functional evidence provides strong support for a model in which the $\mathrm{N}$ - and perhaps also the C-terminus modifies transitions between the open and closed states by direct interactions with the S4-S5 linker that may modify the stability of the activated configuration of the hERG channel voltage sensor. 


\section{SUMMARY}

Despite significant attention, the mechanistic basis of the physiologically and pharmacologically important slow activation and deactivation processes in hERG channels remain unclear. Here, we have taken a comparative approach to evaluate possible mechanisms responsible by discussing the role of sequence divergence in hERG and the archetypal Kv channel, Shaker. Structurally speaking, the available data suggest that the voltage-sensing domain in hERG channels is not significantly different from that in Shaker. Functionally speaking, the intra-voltage-sensing domain chargecharge interactions also seem quite similar in hERG and Shaker channels. This leads the search for the mechanistic basis underlying hERG's unique gating processes elsewhere. Recent evidence suggests that a gating charge transfer center may exist within the voltage sensor domain of hERG, as in Shaker channels, and that key differences in the nature of the charges at the inner and outer margins of the S4 segment are important in regulating gating

\section{REFERENCES}

Aggarwal, S. K., and Mackinnon, R. (1996). Contribution of the S4 segment to gating charge in the shaker $\mathrm{K}^{+}$channel. Neuron 16, 1169-1177.

Alonso-Ron, C., de la Peña, P., Miranda, P., Dominguez, P., and Barros, F. (2008). Thermodynamic and kinetic properties of amino-terminal and S4-S5 loop HERG channel mutants under steady-state conditions. Biophys. J. 94, 3893-3911.

Al-Owais, M., Bracey, K., and Wray, D. (2009). Role of intracellular domains in the function of the herg potassium channel. Eur. Biophys. J. 38, 569-576.

Armstrong, C. M., and Bezanilla, F. (1974). Charge movement associated with the opening and closing of the activation gates of the Na channels. J. Gen. Physiol. 63, 533-552.

Aydar, E., and Palmer, C. (2001). Functional characterization of the Cterminus of the human ether-a-gogo-related gene $\mathrm{K}^{+}$channel (HERG). J. Physiol. (Lond.) 534, 1-14.

Baker, O. S., Larsson, H. P., Mannuzzu, L. M., and Isacoff, E. Y. (1998). Three transmembrane conformations and sequence-dependent displacement of the S4 domain in shaker $\mathrm{K}^{+}$channel gating. Neuron 20, 1283-1294.

Barros, F., Domínguez, P., and de la Peña, P. (2012). Cytoplasmic domains and voltagedependent potassium channel gating. Front. Pharmacol. 3:49. doi:10.3389/fphar.2012.00049

Batulan, Z., Haddad, G. A., and Blunck, R. (2010). An intersubunit interaction between S4-S5 linker and S6 is responsible for the slow off-gating component in shaker $\mathrm{K}^{+}$channels. J. Biol. Chem. 285, 14005-14019.
Bett, G. C., Zhou, Q., and Rasmusson, R. L. (2011). Models of HERG gating. Biophys. J. 101, 631-642.

Bezanilla, F. (2000). The voltage sensor in voltage-dependent ion channels. Physiol. Rev. 80, 555-592.

Bezanilla, F., Perozo, E., and Stefani, E. (1994). Gating of shaker $\mathrm{K}^{+}$channels: II. The components of gating currents and a model of channel activation. Biophys. J. 66, 1011-1021.

Börjesson, S. I., and Elinder, F. (2008). Structure, function, and modification of the voltage sensor in voltagegated ion channels. Cell Biochem. Biophys. 52, 149-174.

Campos, F. V., Chanda, B., Roux, B., and Bezanilla, F. (2007). Two atomic constraints unambiguously position the S4 segment relative to S1 and S2 segments in the closed state of shaker $\mathrm{K}$ channel. Proc. Natl. Acad. Sci. U.S.A. 104, 7904-7909.

Catterall, W. A. (2010). Ion channel voltage sensors: structure, function, and pathophysiology. Neuron 67, 915-928.

Cha, A., and Bezanilla, F. (1997). Characterizing voltage-dependent conformational changes in the shaker $\mathrm{K}^{+}$channel with fluorescence. $\mathrm{Neu}$ ron 19, 1127-1140.

Chanda, B., and Bezanilla, F. (2008). A common pathway for charge transport through voltage-sensing domains. Neuron 57, 345-351.

Chen, J., Mitcheson, J. S., TristaniFirouzi, M., Lin, M., and Sanguinetti, M. C. (2001). The S4-S5 linker couples voltage sensing and activation Acad. Sci. U.S.A. 98, 11277-11282.

Chen, X., Wang, Q., Ni, F., and Ma, J. (2010). Structure of the full-length shaker potassium channel Kv1.2 by of pacemaker channels. Proc. Natl.

differences between these two channels. In addition, sequence divergence at the level of the S4-S5 linker also contributes to the slow gating in hERG channels. Data suggest that $\mathrm{S} 4-\mathrm{S} 5$ linker flexibility changes may be a key determinant of hERG gating, enabling integration of cytoplasmic domain interactions with the stability of the voltage sensor domain in resting and activated states. Although many questions remain concerning the intriguingly unique gating characteristics of hERG channels, these new observations provide a tantalizing view of how $\mathrm{Kv}$ channel functional diversity may have evolved via subtle alterations in the primary sequence.

\section{ACKNOWLEDGMENTS}

This work was supported by a Heart and Stroke Foundation of Canada New Investigator Award and a Michael Smith Foundation for Health Research Career Scholar Award held by Tom W. Claydon.

normal-mode-based X-ray crystallographic refinement. Proc. Natl. Acad. Sci. U.S.A. 107, 11352-11357.

Cheng, Y. M., Hull, C. M., Niven, C. M., Allard, C. R., and Claydon, T. W. (2012). Molecular determinants of voltage-dependent gating in hERG potassium channels. Biophys. J. 102, 329a.

Clancy, C. E., and Rudy, Y. (2001). Cellular consequences of HERG mutations in the long QT syndrome: precursors to sudden cardiac death. Cardiovasc. Res. 50, 301-313.

de la Peña, P., Alonso-Ron, C., Machin, A., Fernández-Trillo, J., Carretero, L., Dominguez, P., and Barros, F. (2011). Demonstration of physical proximity between the $\mathrm{N}$ terminus and the S4-S5 linker of the human ether-a-go-go-related gene (hERG) potassium channel. J. Biol. Chem. 286, 19065-19075.

del Camino, D., Holmgren, M., Liu, Y. and Yellen, G. (2000). Blocker protection in the pore of a voltagegated $\mathrm{K}^{+}$channel and its structural implications. Nature 403, 321-325.

del Camino, D., and Yellen, G. (2001) Tight steric closure at the intracellular activation gate of a voltage-gated $\mathrm{K}^{+}$channel. Neuron 32, 649-656.

Ding, S., and Horn, R. (2002). Tail end of the S6 segment: role in permeation in shaker potassium channels. J. Gen. Physiol. 120, 87-97.

Ding, S., and Horn, R. (2003). Effect of S6 tail mutations on charge movement in shaker potassium channels. Biophys. J. 84, 295-305.

Doyle, D. A., Morais-Cabral, J. H., Pfuetzner, R. A., Kuo, A., Gulbis, J. M., Cohen, S. L., Chait, B. T., and Mackinnon, R. (1998). The structure of the potassium channel: molecular basis of $\mathrm{K}^{+}$conduction and selectivity. Science 280, 69-77.

Durell, S. R., Hao, Y., and Guy, H. R. (1998). Structural models of the transmembrane region of voltagegated and other $\mathrm{K}^{+}$channels in open, closed, and inactivated conformations. J. Struct. Biol. 121, 263-284.

Elliott, D. J., Dondas, N. Y., Munsey, T. S., and Sivaprasadarao, A. (2009). Movement of the S4 segment in the hERG potassium channel during membrane depolarization. Mol. Membr. Biol. 26, 435-447.

Es-Salah-Lamoureux, Z., Fougere, R., Xiong, P. Y., Robertson, G. A., and Fedida, D. (2010). Fluorescencetracking of activation gating in human ERG channels reveals rapid S4 movement and slow pore opening. PLoS ONE 5, e10876. doi:10.1371/journal.pone.0010876

Faure, E., McGuire, H., Marsolais, M., and Blunck, R. (2012). Movement of the S4-S5 linker of KvAP during gating. Biophys. J. 102, 13a.

Fedida, D., and Hesketh, J. C. (2001). Gating of voltage-dependent potassium channels. Prog. Biophys. Mol. Biol. 75, 165-199.

Fernandez, D., Ghanta, A., Kauffman, G. W., and Sanguinetti, M. C. (2004). Physicochemical features of the hERG channel drug binding site. J. Biol. Chem. 279, 10120-10127.

Fernández-Trillo, J., Barros, F., Machín, A., Carretero, L., Domínguez, P., and de la Peña, P. (2011). Molecular determinants of interactions between the $\mathrm{N}$-terminal domain and the transmembrane core that modulate hERG $\mathrm{K}^{+}$channel gating. PLoS ONE 6, e24674. doi:10.1371/journal.pone. 0024674 
Ferrer, T., Rupp, J., Piper, D. R., and Tristani-Firouzi, M. (2006). The S4S5 linker directly couples voltage sensor movement to the activation gate in the human ether-a'-go-gorelated gene (hERG) $\mathrm{K}^{+}$channel. J. Biol. Chem. 281, 12858-12864.

Gayen, S., Li, Q., and Kang, C. (2012). The solution structure of the S4S5 linker of the hERG potassium channel. J. Pept. Sci. 18, 140-145.

Gilly, W. F., and Armstrong, C. M. (1980). Gating current and potassium channels in the giant axon of the squid. Biophys. J. 29, 485-492.

Gómez-Varela, D., de la Peña, P., García, J., Giráldez, T., and Barros, F. (2002). Influence of amino-terminal structures on kinetic transitions between several closed and open states in human erg $\mathrm{K}^{+}$channels. J. Membr. Biol. 187, 117-133.

Gustina, A. S., and Trudeau, M. C. (2009). A recombinant N-terminal domain fully restores deactivation gating in N-truncated and long QT syndrome mutant hERG potassium channels. Proc. Natl. Acad. Sci. U.S.A. 106, 13082-13087.

Gustina, A. S., and Trudeau, M. C. (2011). hERG potassium channel gating is mediated by $\mathrm{N}$ - and $\mathrm{C}$ terminal region interactions. J. Gen. Physiol. 137, 315-325.

Hackos, D. H., Chang, T. H., and Swartz, K. J. (2002). Scanning the intracellular S6 activation gate in the shaker $\mathrm{K}^{+}$channel. J. Gen. Physiol. 119, 521-532.

Hodgkin, A. L., and Huxley, A. F. (1952). A quantitative description of membrane current and its application to conduction and excitation in nerve. J. Physiol. (Lond.) 117, 500-544.

Holmgren, M., Jurman, M. E., and Yellen, G. (1996). N-type inactivation and the S4-S5 region of the shaker $\mathrm{K}^{+}$channel. J. Gen. Physiol. 108, 195-206.

Isacoff, E. Y., Jan, Y. N., and Jan, L. Y. (1991). Putative receptor for the cytoplasmic inactivation gate in the shaker $\mathrm{K}^{+}$channel. Nature 353, 86-90.

Jiang, Y., Lee, A., Chen, J., Cadene, M., Chait, B. T., and MacKinnon, R. (2002a). The open pore conformation of potassium channels. Nature 417, 523-526.

Jiang, Y., Lee, A., Chen, J., Cadene, M., Chait, B. T., and MacKinnon, R. (2002b). Crystal structure and mechanism of a calciumgated potassium channel. Nature 417, 515-522.

Jiang, Y., Lee, A., Chen, J., Ruta, V., Cadene, M., Chait, B. T., and MacKinnon, R. (2003). X-ray structure of a voltage-dependent $\mathrm{K}^{+}$channel. Nature 423, 33-41.

Kanevsky, M., and Aldrich, R. W. (1999). Determinants of voltage-dependent gating and open-state stability in the S5 segment of shaker potassium channels. J. Gen. Physiol. 114, 215-242.

Keynes, R. D., and Elinder, F. (1999). The screw-helical voltage gating of ion channels. Proc. Biol. Sci. 266, 843-852.

Khalili-Araghi, F., Jogini, V., YarovYarovoy, V., Tajkhorshid, E., Roux, B., and Schulten, K. (2010). Calculation of the gating charge for the Kv1.2 voltage-activated potassium channel. Biophys J 98, 2189-2198.

Kitaguchi, T., Sukhareva, M., and Swartz, K. J. (2004). Stabilizing the closed S6 gate in the shaker Kv channel through modification of a hydrophobic seal. J. Gen. Physiol. 124, 319-332.

Kurata, H. T., and Fedida, D. (2006). A structural interpretation of voltagegated potassium channel inactivation. Prog. Biophys. Mol. Biol. 92, 185-208.

Labro, A. J., Raes, A. L., Grottesi, A., Van, H. D., Sansom, M. S., and Snyders, D. J. (2008). Kv channel gating requires a compatible S4-S5 linker and bottom part of S6, constrained by non-interacting residues. J. Gen. Physiol. 132, 667-680.

Lacroix, J. J., and Bezanilla, F. (2011). Control of a final gating charge transition by a hydrophobic residue in the S2 segment of a $\mathrm{K}^{+}$channel voltage sensor. Proc. Natl. Acad. Sci. U.S.A. 108, 6444-6449.

Larsson, H. P., Baker, O. S., Dhillon, D. S., and Isacoff, E. Y. (1996). Transmembrane movement of the shaker $\mathrm{K}^{+}$channel S4. Neuron 16, 387-397.

Ledwell, J. L., and Aldrich, R. W. (1999). Mutations in the $\mathrm{S} 4$ region isolate the final voltage-dependent cooperative step in potassium channel activation. J. Gen. Physiol. 113, 389-414.

Li, Q., Gayen, S., Chen, A. S., Huang, Q., Raida, M., and Kang, C. (2010). NMR solution structure of the $\mathrm{N}$ terminal domain of hERG and its interaction with the S4-S5 linker. Biochem. Biophys. Res. Commun. 403, 126-132.

Liman, E. R., Hess, P., Weaver, F., and Koren, G. (1991). Voltage-sensing residues in the $\mathrm{S} 4$ region of a mammalian $\mathrm{K}^{+}$channel. Nature 353, 752-756.

Lin, M. C., Hsieh, J. Y., Mock, A. F., and Papazian, D. M. (2011). R1 in the shaker S4 occupies the gating charge transfer center in the resting state. $J$. Gen. Physiol. 138, 155-163.
Lin, M. C., and Papazian, D. M. (2007). Differences between ion binding to eag and HERG voltage sensors contribute to differential regulation of activation and deactivation gating. Channels (Austin) 1, 429-437.

Liu, J., Zhang, M., Jiang, M., and Tseng, G. N. (2003). Negative charges in the transmembrane domains of the HERG $\mathrm{K}$ channel are involved in the activation- and deactivationgating processes. J. Gen. Physiol. 121, 599-614.

Liu, S., Rasmusson, R. L., Campbell, D. L., Wang, S., and Strauss, H. C. (1996). Activation and inactivation kinetics of an E-4031-sensitive current from single ferret atrial myocytes. Biophys. J. 70, 2704-2715.

Liu, Y., Holmgren, M., Jurman, M. E. and Yellen, G. (1997). Gated access to the pore of a voltage-dependent $\mathrm{K}^{+}$channel. Neuron 19, 175-184.

Long, S. B., Campbell, E. B., and MacKinnon, R. (2005a). Crystal structure of a mammalian voltage-dependent shaker family $\mathrm{K}^{+}$channel. Science 309, 897-903

Long, S. B., Campbell, E. B., and MacKinnon, R. (2005b). Voltage sensor of Kv1.2: structural basis of electromechanical coupling. Science 309 903-908.

Long, S. B., Tao, X., Campbell, E. B. and MacKinnon, R. (2007). Atomic structure of a voltage-dependent $\mathrm{K}^{+}$ channel in a lipid membrane-like environment. Nature 450, 376-382.

Loots, E., and Isacoff, E. Y. (1998). Protein rearrangements underlying slow inactivation of the shaker $\mathrm{K}^{+}$channel. J. Gen. Physiol. 112, 377-389.

Lu, Z., Klem, A. M., and Ramu, Y. (2002). Coupling between voltage sensors and activation gate in voltage-gated $\mathrm{K}^{+}$channels. J. Gen. Physiol. 120, 663-676.

Mannuzzu, L. M., Moronne, M. M., and Isacoff, E. Y. (1996). Direct physical measure of conformational rearrangement underlying potassium channel gating. Science 271, 213-216.

Mazhari, R., Greenstein, J. L., Winslow, R. L., Marban, E., and Nuss, H. B. (2001). Molecular interactions between two long-QT syndrome gene products, HERG and KCNE2, rationalized by in vitro and in silico analysis. Circ. Res. 89, 33-38.

McCormack, K., Joiner, W. J., and Heinemann, S. H. (1994). A characterization of the activating structural rearrangements in voltagedependent shaker $\mathrm{K}^{+}$channels. Neuron 12, 301-315.

McCormack, K., Tanouye, M. A., Iverson, L. E., Lin, J. W., Ramaswami, M.,
McCormack, T., Campanelli, J. T., Mathew, M. K., and Rudy, B. (1991). A role for hydrophobic residues in the voltage-dependent gating of Shaker $\mathrm{K}^{+}$channels. Proc. Natl. Acad. Sci. U.S.A. 88, 2931-2935.

Morais Cabral, J. H., Lee, A., Cohen, S. L., Chait, B. T., Li, M., and Mackinnon, R. (1998). Crystal structure and functional analysis of the HERG potassium channel $\mathrm{N}$ terminus: a eukaryotic PAS domain. Cell 95, 649-655.

Muskett, F. W., Thouta, S., Thomson, S. J., Bowen, A., Stansfeld, P. J., and Mitcheson, J. S. (2011). Mechanistic insight into human ether-a-go-go-related gene (hERG) $\mathrm{K}^{+}$channel deactivation gating from the solution structure of the EAG domain. J. Biol. Chem. 286, 6184-6191.

Ng, C. A., Hunter, M. J., Perry, M. D., Mobli, M., Ke, Y., Kuchel, P. W., King, G. F., Stock, D., and Vandenberg, J. I. (2011). The N-terminal tail of hERG contains an amphipathic alpha-helix that regulates channel deactivation. PLOS ONE 6, e16191. doi:10.1371/journal.pone.0016191

Ng, C. A., Perry, M. D., Tan, P. S., Hill, A. P., Kuchel, P. W., and Vandenberg, J. I. (2012). The s4-s5 linker acts as a signal integrator for HERG $\mathrm{k}$ channel activation and deactivation gating. PLoS ONE 7, e31640. doi:10.1371/journal.pone.0031640

Nishizawa, M., and Nishizawa, K. (2008). Molecular dynamics simulation of $\mathrm{Kv}$ channel voltage sensor helix in a lipid membrane with applied electric field. Biophys. J. 95, 1729-1744.

Nishizawa, M., and Nishizawa, K. (2009). Coupling of S4 helix translocation and S6 gating analyzed by molecular-dynamics simulations of mutated Kv channels. Biophys. J. 97, 90-100.

Noceti, F., Baldelli, P., Wei, X., Qin, N., Toro, L., Birnbaumer, L., and Stefani, E. (1996). Effective gating charges per channel in voltage-dependent $\mathrm{K}^{+}$and $\mathrm{Ca}^{+}$channels. J. Gen. Physiol. 108, 143-155.

Noda, M., Shimizu, S., Tanabe, T., Takai, T., Kayano, T., Ikeda, T., Takahashi, H., Nakayama, H., Kanaoka, Y., Minamino, N., Kangawa, K., Matsuo, H., Raftery, M., Hirose, T., Inayama, S., Hayashida, H., Miyata, T., and Numa, S. (1984). Primary structure of electrophorus electricus sodium channel deduced from cDNA sequence. Nature 312, 121-127.

Oehmen, C. S., Giles, W. R., and Demir, S. S. (2002). Mathematical model of 
the rapidly activating delayed rectifier potassium current $\mathrm{I}(\mathrm{Kr})$ in rabbit sinoatrial node. J. Cardiovasc. Electrophysiol. 13, 1131-1140.

Ohlenschlager, O., Hojo, H., Ramachandran, R., Gorlach, M., and Haris, P. I. (2002). Three-dimensional structure of the S4-S5 segment of the shaker potassium channel. Biophys. J. 82, 2995-3002.

Papazian, D. M., and Bezanilla, F. (1997). How does an ion channel sense voltage? News Physiol. Sci. 12, 203-210.

Papazian, D. M., Schwarz, T. L., Tempel, B. L., Jan, Y. N., and Jan, L. Y. (1987). Cloning of genomic and complementary DNA from shaker, a putative potassium channel gene from Drosophila. Science 237, 749-753.

Papazian, D. M., Shao, X. M., Seoh, S. A., Mock, A. F., Huang, Y., and Wainstock, D. H. (1995). Electrostatic interactions of S4 voltage sensor in shaker $\mathrm{K}^{+}$channel. Neuron 14 , 1293-1301.

Papazian, D. M., Timpe, L. C., Jan, Y. N., and Jan, L. Y. (1991). Alteration of voltage-dependence of shaker potassium channel by mutations in the $\mathrm{S} 4$ sequence. Nature 349, 305-310.

Pathak, M. M., Yarov-Yarovoy, V., Agarwal, G., Roux, B., Barth, P., Kohout, S., Tombola, F., and Isacoff, E. Y. (2007). Closing in on the resting state of the shaker $\mathrm{K}^{+}$channel. Neuron 56, 124-140.

Piper, D. R., Hinz, W. A., Tallurri, C. K., Sanguinetti, M. C., and TristaniFirouzi, M. (2005). Regional specificity of human ether-a'-go-gorelated gene channel activation and inactivation gating. J. Biol. Chem. 280, 7206-7217.

Piper, D. R., Rupp, J., Sachse, F. B., Sanguinetti, M. C., and Tristani-Firouzi, M. (2008). Cooperative interactions between R531 and acidic residues in the voltage sensing module of hERG1 channels. Cell. Physiol. Biochem. 21, 37-46.

Piper, D. R., Varghese, A., Sanguinetti, M. C., and Tristani-Firouzi, M. (2003). Gating currents associated with intramembrane charge displacement in HERG potassium channels. Proc. Natl. Acad. Sci. U.S.A. 100, 10534-10539.

Pless, S. A., Galpin, J. D., Niciforovic, A. P., and Ahern, C. A. (2011). Contributions of counter-charge in a potassium channel voltagesensor domain. Nat. Chem. Biol. 7, 617-623.

Saenen, J. B., Labro, A. J., Raes, A., and Snyders, D. J. (2006). Modulation of HERG gating by a charge cluster in the N-terminal proximal domain. Biophys. J. 91, 4381-4391.

Sanguinetti, M. C., and Tristani-Firouzi, M. (2006). hERG potassium channels and cardiac arrhythmia. Nature 440, 463-469.

Sanguinetti, M. C., and Xu, Q. P. (1999). Mutations of the S4-S5 linker alter activation properties of HERG potassium channels expressed in Xenopus oocytes. J. Physiol. (Lond.) 514, 667-675.

Schonherr, R., and Heinemann, S. H. (1996). Molecular determinants for activation and inactivation of HERG, a human inward rectifier potassium channel. J. Physiol. (Lond.) 493, 635-642.

Schoppa, N. E., McCormack, K., Tanouye, M. A., and Sigworth, F. J. (1992). The size of gating charge in wild-type and mutant shaker potassium channels. Science 255, 1712-1715.

Schoppa, N. E., and Sigworth, F. J. (1998a). Activation of shaker potassium channels. III. An activation gating model for wild-type and V2 mutant channels. J. Gen. Physiol. 111, 313-342.

Schoppa, N. E., and Sigworth, F. J. (1998b). Activation of shaker potassium channels. II. Kinetics of the V2 mutant channel. J. Gen. Physiol. 111, 295-311.

Schow, E. V., Freites, J. A., Gogna, K., White, S. H., and Tobias, D. J. (2010). Down-state model of the voltagesensing domain of a potassium channel. Biophys. J. 98, 2857-2866.

Seoh, S. A., Sigg, D., Papazian, D. M., and Bezanilla, F. (1996). Voltage-sensing residues in the S2 and S4 segments of the shaker $\mathrm{K}^{+}$channel. Neuron 16 , 1159-1167.

Silverman, W. R., Roux, B., and Papazian, D. M. (2003). Structural basis of two-stage voltage-dependent activation in $\mathrm{K}^{+}$channels. Proc. Natl. Acad. Sci. U.S.A. 100, 2935-2940.

Smith, P. L., and Yellen, G. (2002). Fast and slow voltage sensor movements in HERG potassium channels. J. Gen. Physiol. 119, 275-293.

Spector, P. S., Curran, M. E., Zou, A., Keating, M. T., and Sanguinetti, M. C. (1996). Fast inactivation causes rectification of the IKr channel. J. Gen. Physiol. 107, 611-619.

Starace, D. M., and Bezanilla, F. (2001). Histidine scanning mutagenesis of basic residues of the S4 segment of the shaker $\mathrm{K}^{+}$channel. J. Gen. Physiol. 117, 469-490.

Starace, D. M., and Bezanilla, F. (2004). A proton pore in a potassium channel voltage sensor reveals a focused electric field. Nature 427, 548-553.
Starace, D. M., Stefani, E., and Bezanilla, F. (1997). Voltage-dependent proton transport by the voltage sensor of the shaker $\mathrm{K}^{+}$channel. Neuron 19, 1319-1327.

Subbiah, R. N., Clarke, C. E., Smith, D. J., Zhao, J., Campbell, T. J., and Vandenberg, J. I. (2004). Molecular basis of slow activation of the human ether-a-go-go related gene potassium channel. J. Physiol. (Lond.) 558, 417-431.

Subbiah, R. N., Kondo, M., Campbell, T. J., and Vandenberg, J. I. (2005). Tryptophan scanning mutagenesis of the HERG $\mathrm{K}^{+}$channel: the S4 domain is loosely packed and likely to be lipid exposed. J. Physiol. (Lond.) 569, 367-379.

Subbotina, J., Yarov-Yarovoy, V., LeesMiller, J., Durdagi, S., Guo, J., Duff, H. J., and Noskov, S. Y. (2010). Structural refinement of the hERG1 pore and voltage-sensing domains with ROSETTA-membrane and molecular dynamics simulations. Proteins 78, 2922-2934.

Tao, X., Lee, A., Limapichat, W. Dougherty, D. A., and Mackinnon, R. (2010). A gating charge transfer center in voltage sensors. Science 328, 67-73.

Tiwari-Woodruff, S. K., Lin, M. A., Schulteis, C. T., and Papazian, D. M. (2000). Voltage-dependent structural interactions in the Shaker $\mathrm{K}^{+}$channel. J. Gen. Physiol. 115, 123-138.

Tiwari-Woodruff, S. K., Schulteis, C. T., Mock, A. F., and Papazian, D. M. (1997). Electrostatic interactions between transmembrane segments mediate folding of Shaker $\mathrm{K}^{+}$channel subunits. Biophys. J. 72, 1489-1500.

Tombola, F., Pathak, M. M., and Isacoff, E. Y. (2005). Voltagesensing arginines in a potassium channel permeate and occlude cation-selective pores. Neuron 45, 379-388.

Tristani-Firouzi, M., Chen, J., and Sanguinetti, M. C. (2002). Interactions between S4-S5 linker and S6 transmembrane domain modulate gating of HERG K ${ }^{+}$channels. J. Biol. Chem. 277, 18994-19000.

Trudeau, M. C., Warmke, J. W., Ganetzky, B., and Robertson, G. A. (1995). HERG, a human inward rectifier in the voltage-gated potassium channel family. Science 269, 92-95.

Van Slyke, A. C., Rezazadeh, S., Snopkowski, M., Shi, P., Allard, C. R., and Claydon, T. W. (2010). Mutations within the S4-S5 linker alter voltage sensor constraints in hERG $\mathrm{K}^{+}$ channels. Biophys. J. 99, 2841-2852.
Vargas, E., Bezanilla, F., and Roux, B. (2011). In search of a consensus model of the resting state of a voltage-sensing domain. Neuron 72 , 713-720.

Viloria, C. G., Barros, F., Giráldez, T., Gómez-Varela, D., and de la Peña, P. (2000). Differential effects of amino-terminal distal and proximal domains in the regulation of human erg $\mathrm{K}^{+}$channel gating. Biophys. J. 79, 231-246.

Wang, J., Myers, C. D., and Robertson, G. A. (2000). Dynamic control of deactivation gating by a soluble amino-terminal domain in HERG $\mathrm{K}^{+}$channels. J. Gen. Physiol. 115, 749-758.

Wang, J., Trudeau, M. C., Zappia, A. M., and Robertson, G. A. (1998). Regulation of deactivation by an amino terminal domain in human ether-a-go-go-related gene potassium channels. J. Gen. Physiol. 112, 637-647.

Wang, M. H., Yusaf, S. P., Elliott, D. J., Wray, D., and Sivaprasadarao, A. (1999). Effect of cysteine substitutions on the topology of the S4 segment of the shaker potassium channel: implications for molecular models of gating. J. Physiol. (Lond.) 521(Pt 2), 315-326.

Wang, S., Liu, S., Morales, M. J., Strauss, H. C., and Rasmusson, R. L. (1997). A quantitative analysis of the activation and inactivation kinetics of HERG expressed in Xenopus oocytes. J. Physiol. (Lond.) 502(Pt 1), 45-60.

Wynia-Smith, S. L., Gillian-Daniel, A. L., Satyshur, K. A., and Robertson, G. A (2008). hERG gating microdomains defined by $\mathrm{S} 6$ mutagenesis and molecular modeling. J. Gen. Physiol. 132, 507-520.

Yarov-Yarovoy, V., Baker, D., and Catterall, W. A. (2006). Voltage sensor conformations in the open and closed states in ROSETTA structural models of $\mathrm{K}^{+}$channels. Proc. Natl. Acad. Sci. U.S.A. 103, 7292-7297.

Yusaf, S. P., Wray, D., and Sivaprasadarao, A. (1996). Measurement of the movement of the S4 segment during the activation of a voltage-gated potassium channel. Pflugers Arch. 433, 91-97.

Zagotta, W. N., Hoshi, T., and Aldrich, R. W. (1994a). Shaker potassium channel gating. III: evaluation of kinetic models for activation. J. Gen. Physiol. 103, 321-362.

Zagotta, W. N., Hoshi, T., Dittman, J., and Aldrich, R. W. (1994b). Shaker potassium channel gating. II: transitions in the activation pathway. $J$. Gen. Physiol. 103, 279-319. 
Zhang, M., Liu, J., Jiang, M., Wu, D. M., Sonawane, K., Guy, H. R., and Tseng, G. N. (2005). Interactions between charged residues in the transmembrane segments of the voltage-sensing domain in the hERG channel. J. Membr. Biol. 207, 169-181.

Zhang, M., Liu, J., and Tseng, G. N. (2004). Gating charges in the activation and inactivation processes of the HERG channel. J. Gen. Physiol. 124, 703-718.

Conflict of Interest Statement: The authors declare that the research was conducted in the absence of any commercial or financial relationships that could be construed as a potential conflict of interest.
Received: 16 March 2012; accepted: 16 April 2012; published online: 08 May 2012.

Citation: Cheng YM and Claydon TW (2012) Voltage-dependent gating of hERG potassium channels. Front. Pharmacol. 3:83. doi: 10.3389/fphar.2012.00083

This article was submitted to Frontiers in Pharmacology of Ion Channels and
Channelopathies, a specialty of Frontiers in Pharmacology.

Copyright (c) 2012 Cheng and Claydon.

This is an open-access article distributed under the terms of the Creative Commons Attribution Non Commercial License, which permits non-commercial use, distribution, and reproduction in other forums, provided the original authors and source are credited. 